\title{
A 3-NODE CO-ROTATIONAL TRIANGULAR ELASTO-PLASTIC SHELL ELEMENT USING VECTORIAL ROTATIONAL VARIABLES
}

\author{
Zhongxue Li ${ }^{1, *}$, Bassam A. Izzuddin ${ }^{2}$, Loc Vu-Quoc ${ }^{3}$, Zihan Rong ${ }^{4}$ and Xin Zhuo ${ }^{4}$ \\ 1, * Department of Civil Engineering, Zhejiang University, China \\ 2 Department of Civil and Environmental Engineering, Imperial College London, United Kingdom \\ ${ }^{3}$ Department of Mechanical and Aerospace Engineering, University of Florida, USA \\ ${ }^{4}$ Department of Civil Engineering, Zhejiang University, PR China \\ *(Corresponding author: E-mail: lizx19993@zju.edu.cn)
}

Received: 21 March 2016; Revised: 31 July 2016; Accepted: 3 August 2016

\begin{abstract}
A 3-node co-rotational triangular elasto-plastic shell element is developed. The local coordinate system of the element employs a zero-'macro spin' framework at the macro element level, thus reducing the material spin over the element domain, and resulting in an invariance of the element tangent stiffness matrix to the order of the node numbering. The two smallest components of each nodal orientation vector are defined as rotational variables, achieving the desired additive property for all nodal variables in a nonlinear incremental solution procedure. Different from other existing co-rotational finite-element formulations, both element tangent stiffness matrices in the local and global coordinate systems are symmetric owing to the commutativity of the nodal variables in calculating the second derivatives of strain energy with respect to the local nodal variables and, through chain differentiation with respect to the global nodal variables. For elasto-plastic analysis, the Maxwell-Huber-Hencky-von Mises yield criterion is employed together with the backward-Euler return-mapping method for the evaluation of the elasto-plastic stress state, where a consistent tangent modulus matrix is used. Assumed membrane strains and assumed shear strains---calculated respectively from the edge-member membrane strains and the edge-member transverse shear strains---are employed to overcome locking problems, and the residual bending flexibility is added to the transverse shear flexibility to improve further the accuracy of the element. The reliability and convergence of the proposed 3-node triangular shell element formulation are verified through two elastic plate patch tests as well as three elastic and three elasto-plastic plate/shell problems undergoing large displacements and large rotations.
\end{abstract}

Keywords: Co-rotational approach, elasto-plasticity, triangular shell element, assumed strain, vectorial rotational variable, zero-'macro spin'

DOI: $10.18057 /$ IJASC.2017.13.3.2

\section{INTRODUCTION}

Shell structures with different geometry, thickness, loading and boundary conditions are widely applied in engineering practice. Shell finite elements play an important role in analyzing, designing and optimizing shell structures. An optimal shell element should (1) be applicable to arbitrary shell geometries for both thick and thin structures, (2) provide accurate displacements and stresses with low computational cost, and (3) be robust with low sensitivity to element distortion [1-7]. Formulations for flat 3-node triangular shell elements are simpler, and computationally more efficient, than for curved triangular elements with more than 3 nodes. Furthermore, 3-node triangular mesh is the most robust and efficient option of mesh generation. As a result, reliable and computationally efficient 3-node triangular shell elements have important applications in modeling shell structures with arbitrary and complex geometries [8].

A 3-node triangular shell element can be formulated by combining a membrane element and a bending element [9-12], or by relying on three-dimensional continuum mechanics with the Reissner-Mindlin kinematic hypothesis and the plane-stress assumption [8,13]. Existing 3-node triangular shell elements can be categorized into 4 types: Type 1 with only 3 displacement 
degrees-of-freedom (dofs) per node [14-22]; Type 2 with 3 displacement dofs and 2 rotational dofs per node [23-25]; Type 3 with 3 displacement dofs at the vertices and the rotational dofs at side nodes [26-27]; Type 4 with 3 displacement dofs and 3 rotational dofs per node [28-33]. Most existing 3-node rotation-free triangular shell elements are based on the Kirchhoff assumption, ignoring shear deformation, can therefore only be employed in modeling thin plate and shell problems [21]. To extend rotation-free flat 3-node triangular shell elements with transverse shear deformation effects, Zárate and Oñate [21] added two transverse shear angles to the element curvatures which are calculated over the domain formed by the element itself and the three adjacent triangular elements. These two transverse shear angles are considered as rotational degrees of freedom for thick shell problems, and they vanish for thin shell problems. A flat shell element with 5 degrees-of-freedom per corner node can be obtained by combining a conventional triangular membrane element with a standard 9-dof triangular bending element. On the other hand, if several elements of this type sharing the same node are coplanar, it is difficult to achieve inter-element compatibility between membrane and transverse displacements, and the assembled global stiffness matrix is singular in shell analysis due to the absence of in-plane rotation degrees-of-freedom [9-12,34]. In addition, flat shell elements with 5 degrees-of-freedom per node lack proper nodal degrees of freedom to model folded plate/shell structures, making the assembly of elements troublesome [35]. To avoid the singularity of the assembled global stiffness matrix, some researchers defined the displacement degrees-of-freedom at the vertices and the rotational degrees-of-freedom at side nodes of flat shell elements [26-27]. This type of elements cannot, however, be easily matched with other types of elements in modeling of complex structures. Alternatively, some researchers [9-12] added a sixth degree of freedom (the drilling rotation) at each node of flat triangular shell elements by combining a bending element and a membrane element with drilling rotational degrees-of-freedom. Such shell elements are very convenient for engineering applications since no special connection scheme is necessary at the shell edges and intersections, and no particular care needs to be taken when coupling shell and rod elements [36]. Such approach renders, however, the numerical method inconsistent, possibly leading to a poor element convergence [37]. Then, Reissner [38], Hughes and Brezzi [39], and Hughes et al. [40-41] endeavored to develop variational formulations employing independent rotation fields so as to obtain a theoretically sound and practically useful formulation for engineering application.

A major difficulty in developing flat 3-node triangular shell elements is to overcome locking phenomena arising in bending dominated situations without destroying the ability of the procedure to accurately capture membrane dominated and mixed behaviors [7]. In general, flat 3-node triangular shell element cannot well approximate the pure bending displacement field; thus, locking problems are serious and inevitable. As the shell thickness decreases, the convergence of the finite element solution rapidly deteriorates [8]. Researchers developed methods to overcome locking phenomena in flat triangular shell elements, such as the Mixed Interpolation of Tensorial Components method used in the MITC3 element [8], the line integration method used in TRIA3 element [42], the mixed or hybrid formulation [43-45], incompatible displacement methods [46-47], stabilization methods [29,48-54], assumed strain methods [19,28,55], etc.

In the present 3-node elasto-plastic triangular shell element formulation, 3 displacement dofs and 2 vectorial rotational dofs are employed at per node, the Reissner-Mindlin theory is used, in which both the thickness deformation and the normal stress in the direction of the shell thickness are ignored, and an assumed strain method is employed to alleviate the membrane and shear locking phenomena. Here, the assumed membrane strains are calculated from 3 edge-member membrane strains of the 3-node triangular shell element, while the assumed transverse shear strains are evaluated from the 3 edge-member transverse shear strains [42]. In addition, the residual bending flexibility is added to the transverse shear flexibility to improve the accuracy of the element further. For elasto-plastic modeling, the fibre approach which can describe the plastic zone spreading 
process in shell structures undergoing large elastoplastic deformation [56-59] is adopted, the Maxwell-Huber-Hencky-von Mises yield criterion for isotropic hardening case [60-64] is introduced, and the material is assumed to be linear hardening. A backward-Euler return-mapping integration algorithm [60-61] is used to trace the yield surface, and a consistent elasto-plastic tangent modulus matrix is employed. To exclude the influence of element rigid-body rotations from the local displacement field, a zero-'macro spin' co-rotational framework, which can significantly reduce the rigid-body rotations of infinitesimal segments at different material points within the element domain in an aggregate sense [65], is employed for the present element to simplify the stress-strain constitutive relation. Compared to other existing co-rotational element formulations [61-66], the present 3-node triangular elasto-plastic shell element formulation has several features: i) All nodal variables are additive in a nonlinear incremental solution procedure, and as a result, updating the element matrices is simple and efficient; ii) Symmetric element tangent stiffness matrices are obtained in both the local and global coordinate systems, leading to computational efficiency and significant computer storage saving; and iii) The element tangent stiffness matrix is updated using the total values of the nodal variables in an incremental solution procedure, making it advantageous for solving dynamic problems [67-70]. The present 3-node triangular shell element demonstrates satisfying convergence and reliability in solving elastic and elasto-plastic plate/shell problems undergoing large displacements [36, 71-81].

The outline of the paper is as follows. Section 2 presents the kinematics of the 3-node triangular shell element in the local co-rotational system. Section 3 describes the local element response, the assumed-strain procedure used to alleviate locking problems, the calculation of the shear strain correction coefficients corresponding to the residual bending flexibility, and the consideration of elasto-plasticity within the element formulation. Section 4 presents the transformation matrix between the local and global systems, and the element formulation in the global coordinate system. In Section 5, several elastic patch tests and elastic/elasto-plastic plate/shell problems are solved to demonstrate the reliability and convergence of the proposed element formulation. Concluding remarks are given in Section 6.

\section{ELEMENT KINEMATICS IN THE LOCAL CO-ROTATIONAL SYSTEM}

The optimal orientation of the local reference system provides a rotated undeformed configuration such that the relative spin of the material in the current deformed configuration to that in the rotated undeformed configuration is zero. In developing the current triangular shell element formulation, the local co-rotational system is based on zero-spin at the macro element level [65], which reduces the material spin in an aggregate sense over the element domain, and maintains the benefits of invariance to nodal ordering and symmetry of the tangent stiffness matrix. The local and the global Cartesian coordinate systems, and the natural coordinate system, are defined respectively as in Figure 1. The local coordinate system always rotates with the element rigid-body rotation in the deformed configuration, but doesn't deform with the element. 


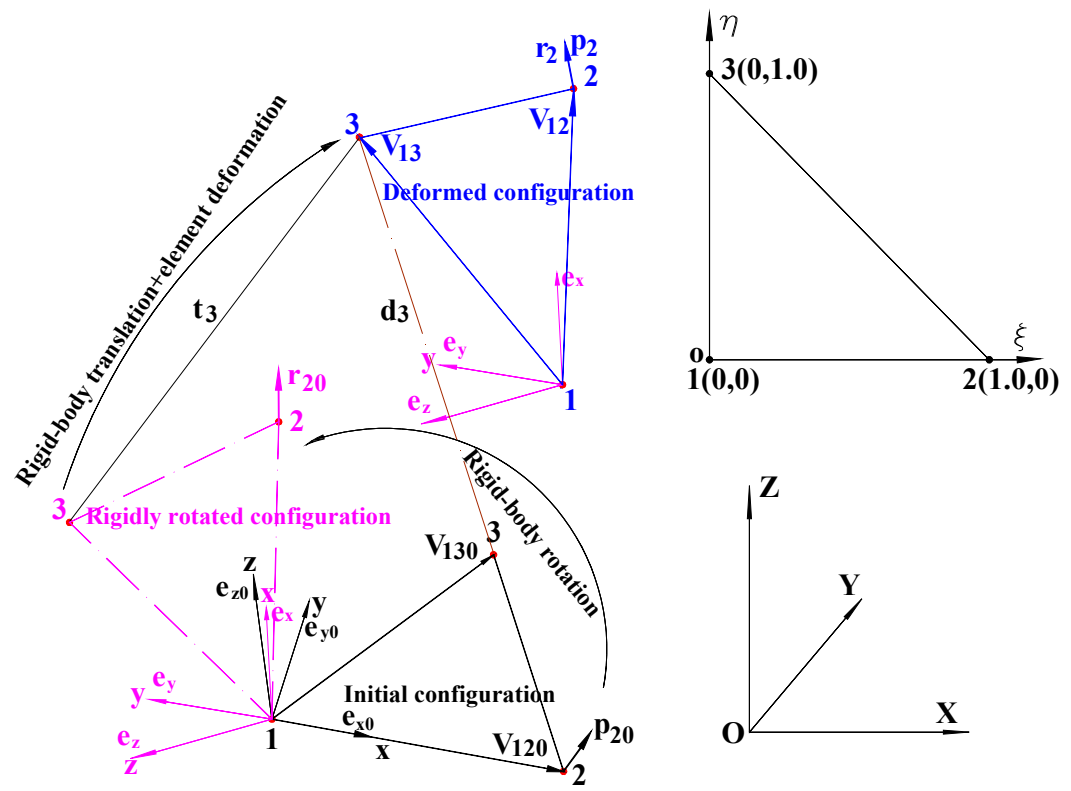

Figure 1. Description of the Co-rotational Framework and the Natural Coordinate System

(Note: The vectors $\mathbf{t}_{3}$ and $\mathbf{r}_{2}$ are associated with the local coordinate system $o-x-y-z$, whereas the vectors $\mathbf{d}_{3}, \mathbf{p}_{20}, \mathbf{p}_{2}, \mathbf{v}_{120}, \mathbf{v}_{12}, \mathbf{v}_{130}, \mathbf{v}_{13}, \mathbf{e}_{\mathrm{x} 0}, \mathbf{e}_{\mathrm{y} 0}, \mathbf{e}_{\mathrm{z} 0}, \mathbf{e}_{\mathrm{x}}, \mathbf{e}_{\mathrm{y}}$ and $\mathbf{e}_{\mathrm{z}}$ are associated with the global coordinate system $\mathrm{O}-\mathrm{X}-\mathrm{Y}-\mathrm{Z}$. .)

In Figure 1, $\mathbf{v}_{i j 0}$ and $\mathbf{v}_{i j}$ are the vectors connecting Node $i$ to Node $j$ in the initial configuration and the current deformed configuration, respectively.

$$
\begin{aligned}
& \mathbf{v}_{i j 0}=\mathbf{X}_{j 0}-\mathbf{X}_{i 0}, \quad i, j=1,2,3 \text { and } i \neq j \\
& \mathbf{v}_{i j}=\mathbf{X}_{j 0}-\mathbf{X}_{i 0}+\mathbf{d}_{j}-\mathbf{d}_{i}, \quad i, j=1,2,3 \text { and } i \neq j
\end{aligned}
$$

where, the vector $\mathbf{X}_{i 0}(i=1,2,3)$ contains the coordinates of Node $i$ in the global coordinate system; the vector $\mathbf{d}_{i}(i=1,2,3)$ represents the displacement of Node $i$ in the global coordinate system. The orientation vector $\mathbf{e}_{x 0}$ of the local $x$-axis as being aligned with edge $1-2$ of the element in the initial configuration,

$\mathbf{e}_{x 0}=\frac{\mathbf{v}_{120}}{\left|\mathbf{v}_{120}\right|}$

and the orientation vector $\mathbf{e}_{z}$ of the local $z$-axis in the current deformed configuration is defined as $\mathbf{e}_{z}=\frac{\mathbf{v}_{12} \times \mathbf{v}_{13}}{\left|\mathbf{v}_{12} \times \mathbf{v}_{13}\right|}$ 


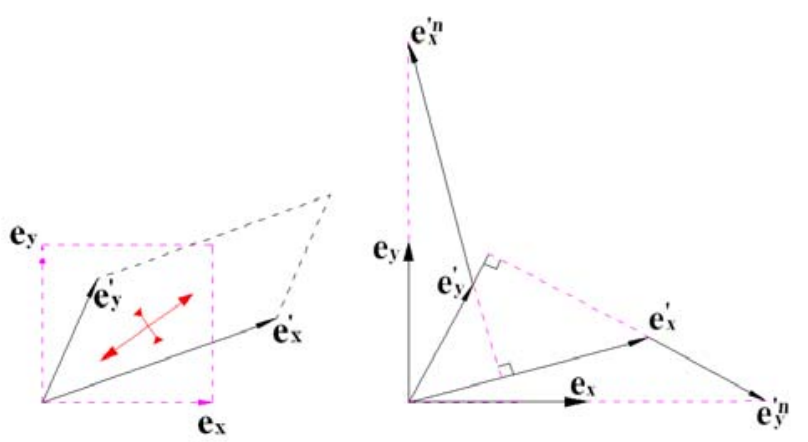

Figure 2. Definition of the Zero-'macro spin' Local Coordinate System

The zero-'macro spin' local coordinate system is generated as follows: A unit square area defined by the two orthogonal unit vectors $\mathbf{e}_{x}$ and $\mathbf{e}_{y}$ (the orientation vectors of the $x$ - and $y$-axes in the deformed configuration, respectively) is subjected to a uniform planar 'stretch' operation in any two orthogonal directions (see Figure 2). Under this planar stretch operation, the vectors $\mathbf{e}_{x}$ and $\mathbf{e}_{y}$ will be transformed into vectors $\mathbf{e}_{x}^{\prime}$ and $\mathbf{e}_{y}^{\prime}$, respectively. The vector $\mathbf{e}_{x}$ is always obtained as the normalized sum of $\mathbf{e}_{x}^{\prime}$ and $\mathbf{e}_{y}^{\prime n}$, where $\mathbf{e}_{y}^{\prime n}$ is a planar rotation of $\mathbf{e}_{y}^{\prime}$ by $-\pi / 2$; likewise, the vector $\mathbf{e}_{y}$ is always obtained as the normalized sum of $\mathbf{e}_{y}^{\prime}$ and $\mathbf{e}_{x}^{n}$, where $\mathbf{e}_{x}^{n}$ is a planar rotation of $\mathbf{e}_{x}^{\prime}$ by $\pi / 2$. Then, the orientation vectors $\mathbf{e}_{x}$ and $\mathbf{e}_{y}$ of the local coordinate system in the current deformed configuration can be obtained from the stretched vectors as:

$\mathbf{e}_{x}=\frac{\mathbf{e}_{x}^{\prime}+\mathbf{e}_{y}^{\prime n}}{\left|\mathbf{e}_{x}^{\prime}+\mathbf{e}_{y}^{\prime n}\right|} \quad \mathbf{e}_{y}^{\prime n}=\mathbf{e}_{y}^{\prime} \times \mathbf{e}_{z}, \quad \mathbf{e}_{y}=\mathbf{e}_{z} \times \mathbf{e}_{x}$

and the stretched vectors $\mathbf{e}_{x}^{\prime}$ and $\mathbf{e}_{y}^{\prime}$ in the current deformed configuration are calculated as

$\mathbf{e}_{x}^{\prime}=a_{x 1} \mathbf{v}_{12}+a_{x 2} \mathbf{v}_{23}$

$\mathbf{e}_{y}^{\prime}=a_{y 1} \mathbf{v}_{12}+a_{y 2} \mathbf{v}_{23}$

Considering that the orientation vectors $\mathbf{e}_{x 0}, \mathbf{e}_{y 0}$ and $\mathbf{e}_{z 0}$ of the local coordinate system in the initial configuration can also be obtained by the same procedure as $\mathbf{e}_{x}, \mathbf{e}_{y}$ and $\mathbf{e}_{z}$ :

$$
\begin{aligned}
& \mathbf{e}_{x 0}^{\prime}=\mathbf{e}_{x 0}=\frac{\mathbf{v}_{120}}{\left|\mathbf{v}_{120}\right|}=a_{x 1} \mathbf{v}_{120}+a_{x 2} \mathbf{v}_{230} \\
& \mathbf{e}_{y 0}^{\prime}=\mathbf{e}_{y 0}=\mathbf{e}_{z 0} \times \mathbf{e}_{x 0}=\frac{\mathbf{v}_{120} \times \mathbf{v}_{230} \times \mathbf{v}_{120}}{\left|\mathbf{v}_{120} \times \mathbf{v}_{230}\right|\left|\mathbf{v}_{120}\right|}=a_{y 1} \mathbf{v}_{120}+a_{y 2} \mathbf{v}_{230}
\end{aligned}
$$

then, the constants used in Eqs. 5a,b can be determined from Eqs. 6a,b as 


$$
\begin{aligned}
& a_{x 1}=\frac{1}{\left|\mathbf{v}_{120}\right|,} \quad a_{x 2}=0 \\
& a_{y 1}=-\frac{\mathbf{e}_{120}^{\mathrm{T}} \mathbf{e}_{230}}{\left|\mathbf{v}_{120}\right| \sqrt{1-\left(\mathbf{e}_{120}^{\mathrm{T}} \mathbf{e}_{230}\right)^{2}}}, \quad a_{y 2}=\frac{1}{\left|\mathbf{v}_{230}\right| \sqrt{1-\left(\mathbf{e}_{120}^{\mathrm{T}} \mathbf{e}_{230}\right)^{2}}}
\end{aligned}
$$

There are 15 degrees of freedom for each element in the local coordinate system. In addition to the three translational displacements, two components of the mid-surface normal vector at each node are defined as vectorial rotational variables. The vector of the local nodal variables is given below

$$
\mathbf{u}_{\mathrm{L}}=\left[\begin{array}{lllllllllllllll}
u_{1} & v_{1} & w_{1} & r_{1, x} & r_{1, y} & u_{2} & v_{2} & w_{2} & r_{2, x} & r_{2, y} & u_{3} & v_{3} & w_{3} & r_{3, x} & r_{3, y}
\end{array}\right]^{\mathrm{T}}
$$

where $u_{i}, v_{i}, w_{i}$ are the three displacement dofs of Node $i$, while $r_{i, x}, r_{i, y}$ are the two vectorial rotational dofs of Node $i$, and are the two components of the mid-surface normal vector $\mathbf{r}_{i}$ in the local coordinate system.

There are 15 degrees of freedom in the global coordinate system. The nodal variable vector is given by

$$
\mathbf{u}_{\mathrm{G}}=\left[\begin{array}{lllllllllllllll}
U_{1} & V_{1} & W_{1} & p_{1, n_{1}} & p_{1, m_{1}} & U_{2} & V_{2} & W_{2} & p_{2, n_{2}} & p_{2, m_{2}} & U_{3} & V_{3} & W_{3} & p_{3, n_{3}} & p_{3, m_{3}}
\end{array}\right]^{\mathrm{T}}
$$

where $U_{i}, V_{i}, W_{i}$ are the three displacement dofs of Node $i$, and $p_{i, n_{i}}, p_{i, m_{i}}$ the vectorial rotational dofs, and are the two smallest components of the mid-surface normal vector $\mathbf{p}_{i}$ at Node $i$ in the $\mathrm{X}, \mathrm{Y}, \mathrm{Z}$ directions of the global coordinate system. This definition avoids ill-conditioning in the resulting equations [82].

The local nodal coordinates are calculated from those in the global coordinate system as follows

$\mathbf{x}_{i 0}=\mathbf{R}_{0} \mathbf{v}_{i 0}$

where $\quad \mathbf{x}_{i 0}^{\mathrm{T}}=\left\lfloor\begin{array}{lll}x_{i 0} & y_{i 0} & z_{i 0}\end{array}\right\rfloor, \mathbf{R}_{0}^{\mathrm{T}}=\left[\begin{array}{ll}\mathbf{e}_{x 0} & \mathbf{e}_{y 0} \\ \mathbf{e}_{z 0}\end{array}\right]$ and $\mathbf{v}_{i 0}=\mathbf{X}_{i 0}-\mathbf{X}_{10}$.

The relationships between the local nodal dofs and the global nodal dofs are given by

$$
\begin{aligned}
& \mathbf{t}_{i}=\mathbf{R}\left(\mathbf{d}_{i}+\mathbf{v}_{i 0}\right)-\mathbf{R}_{0} \mathbf{v}_{i 0} \\
& \boldsymbol{\theta}_{i 0}=\mathbf{R}_{h 0} \mathbf{p}_{i 0} \\
& \boldsymbol{\theta}_{i}=\mathbf{R}_{h} \mathbf{p}_{i}, \quad i=1,2, \ldots, 3
\end{aligned}
$$

where the components of the vector $\mathbf{t}_{i}^{\mathrm{T}}=\left\lfloor\begin{array}{lll}u_{i} & v_{i} & w_{i}\end{array}\right\rfloor$ are the three displacement dofs of Node $i$ in the local coordinate system; the following matrices contain the unit basis vectors $\mathbf{R}_{h 0}^{\mathrm{T}}=\left[\mathbf{e}_{x 0} \mathbf{e}_{y 0}\right] ; \mathbf{R}^{\mathrm{T}}=\left[\begin{array}{ll}\mathbf{e}_{x} & \mathbf{e}_{y} \\ \mathbf{e}_{z}\end{array}\right] ; \mathbf{R}_{h}^{\mathrm{T}}=\left[\mathbf{e}_{x} \mathbf{e}_{y}\right] ;$ the components of the vector $\mathbf{d}_{i}^{\mathrm{T}}=\left[\begin{array}{lll}U_{i} & V_{i} & W_{i}\end{array}\right\rfloor$ are the three displacement dofs of Node $i$ in the global coordinate system; $\mathbf{e}_{x}, \mathbf{e}_{y}$ and $\mathbf{e}_{z}$ are updated 
by using $\mathbf{d}_{i}$ obtained at each iteration of every incremental loading step (referred to Eqs.(1b),(3),(4a-c) and (5a,b) ); the vectors $\boldsymbol{\theta}_{i}$ and $\boldsymbol{\theta}_{i 0}$ are respectively the sub-vectors of the deformed and the initial mid-surface normal vectors $\mathbf{r}_{i}$ and $\mathbf{r}_{i 0}$ at Node $i$ in the local coordinate

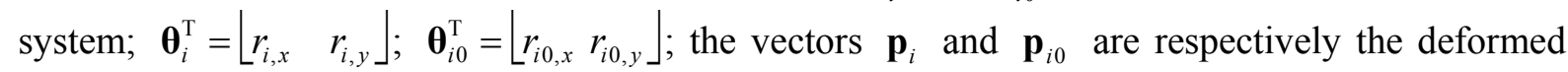
and the initial mid-surface normal vectors at Node $i$ in the global coordinate system. In Eq. (11a), the vectors $\mathbf{R}_{0} \mathbf{v}_{i 0}$ and $\mathbf{R}\left(\mathbf{d}_{i}+\mathbf{v}_{i 0}\right)$ are respectively the local coordinates of Node $i$ in the initial and deformed configurations.

For the description of the geometry and of the displacement field in the 3-node triangular shell element, the interpolation functions used are:

$N_{1}=1-\xi-\eta, \quad N_{2}=\xi, \quad N_{3}=\eta$

In the initial configuration, the local coordinates $\mathbf{x}^{\mathrm{T}}=\left\lfloor\begin{array}{lll}x & y & z\end{array}\right\rfloor$ at any point on the element mid-surface are obtained as:

$\mathbf{x}=\sum_{i=1}^{3} N_{i}(\xi, \eta) \mathbf{x}_{i 0}$

The same shape functions are used to interpolate the displacement and rotation fields, leading to an isoparametric formulation:

$\mathbf{t}=\sum_{i=1}^{3} N_{i}(\xi, \eta) \mathbf{t}_{i}$

$\overline{\mathbf{r}}_{\mathrm{h}}=\sum_{i=1}^{3} N_{i}(\xi, \eta) \boldsymbol{\theta}_{i}$

where the components in the vector $\mathbf{t}^{\mathrm{T}}=\left\lfloor\begin{array}{lll}u & v & w\end{array}\right\rfloor$ are the local displacement fields, and the components in the vector $\overline{\mathbf{r}}_{\mathrm{h}}^{\mathrm{T}}=\left\lfloor\begin{array}{ll}r_{x} & r_{y}\end{array}\right\rfloor$ are the local rotation fields.

The initial mid-surface normal vector at each node of the triangular shell element is obtained by calculating the cross-product of the tangent lines along two natural coordinate axes, i.e.,

$\overline{\mathbf{p}}_{i 0}=\frac{\partial \mathbf{X}_{0}}{\partial \xi} \times\left.\frac{\partial \mathbf{X}_{0}}{\partial \eta}\right|_{\left(\xi_{i}, \eta_{i}\right)}, \quad i=1,2,3$

where $\mathbf{X}_{0}=\sum_{j=1}^{3} N_{j}(\xi, \eta) \mathbf{X}_{j 0}$ represents the global coordinates of a point on the mid-surface of the element; $\left(\xi_{i}, \eta_{i}\right)$ takes the natural coordinates of Node $i$ (referred to Figure 2).

To minimize the discontinuity between the slopes of adjacent elements at Node $i$, the mean value of the normal vectors from the surrounding elements is adopted:

$\mathbf{p}_{i 0}=\frac{\sum^{\overline{\mathbf{p}}_{i 0}} /\left|\overline{\mathbf{p}}_{i 0}\right|}{\left|\sum^{\overline{\mathbf{p}}_{i 0} /} / \overline{\mathbf{p}}_{i 0}\right|}, \quad i=1,2,3$ 
the symbol $\sum$ in Eq. (16) is the summation on the number of elements having the same common Node $i$.

On the other hand, if the true mid-surface of the curved shell is not smooth along the inter-element edges, the normal vector of each shell element must be obtained independently, and three global rotation degrees of freedom would then be required for each node along the edges of slope discontinuity. In general, the two smallest components of one orientation vector and one smaller component of another orientation vector at a node can be selected as global rotational variables, and these vectors can be oriented to three global coordinate axes in the initial configuration or defined as those of the beam element presented in [83-85].

\section{LOCAL ELASTO-PLASTIC ELEMENT FORMULATIONS}

\subsection{Local Response}

The general virtual work statement can be expressed as:

$$
\delta \mathrm{W}=\int_{\mathrm{V}} \boldsymbol{\sigma}^{\mathrm{T}} \delta \boldsymbol{\varepsilon} \mathrm{dV}-\mathbf{f}_{e x t}^{\mathrm{T}} \delta \mathbf{u}_{\mathrm{L}}
$$

where $\mathrm{V}$ is the volume of the element, $\boldsymbol{\sigma}$ the stress vector, $\mathbf{f}_{\text {ext }}$ the external force vector, and $\boldsymbol{\varepsilon}$ the material strain vector; in the case of our shell element, the Reissner-Mindlin theory is adopted, $\boldsymbol{\varepsilon}$ is the Green-Lagrange strain specialized for a shallow curved shell [61,71-72]. For the convenience of calculating the assumed strains below, the material strain vector $\boldsymbol{\varepsilon}$ is split into three parts, $\boldsymbol{\varepsilon}_{m}, z_{l} \boldsymbol{\chi}$ and $\boldsymbol{\gamma}$, representing the membrane strains, bending strains and out-of-plane shear strains, respectively. It follows that Eq. (17) can be rewritten as:

$$
\delta \mathrm{W}=\int_{\mathrm{V}} \boldsymbol{\sigma}^{\mathrm{T}} \delta\left\{\begin{array}{c}
\boldsymbol{\varepsilon}_{m}+z_{l} \boldsymbol{\chi} \\
\boldsymbol{\gamma}
\end{array}\right\} \mathrm{dV}-\mathbf{f}_{\text {ext }}^{\mathrm{T}} \delta \mathbf{u}_{\mathrm{L}}
$$

The internal force vector in the local coordinate system is therefore obtained as:

$$
\mathbf{f}=\mathbf{f}_{e x t}=\int_{\mathrm{V}}\left[\begin{array}{c}
\mathbf{B}_{m}+z_{l} \mathbf{B}_{b} \\
\mathbf{B}_{s}
\end{array}\right]^{\mathrm{T}} \boldsymbol{\sigma} \mathrm{dV}
$$

where $\mathbf{B}_{m}, z_{l} \mathbf{B}_{b}$, and $\mathbf{B}_{s}$ are respectively the first derivatives of the membrane strain vector $\boldsymbol{\varepsilon}_{m}$, the bending strain vector $z_{l} \chi$, and the out-of-plane shear strain vector $\gamma$ with respect to the local nodal variable vector $\mathbf{u}_{\mathrm{L}}$; the details of these matrices are given in Appendix A.

By differentiating the internal force vector with respect to the local nodal variables, the local tangent stiffness matrix of the 3-node triangular shell element is determined as:

$$
\mathbf{k}_{\mathrm{T}}=\int_{\mathrm{V}}\left[\begin{array}{c}
\mathbf{B}_{m}+z_{l} \mathbf{B}_{b} \\
\mathbf{B}_{s}
\end{array}\right]^{\mathrm{T}} \mathbf{D}_{\mathrm{T}}\left[\begin{array}{c}
\mathbf{B}_{m}+z_{l} \mathbf{B}_{b} \\
\mathbf{B}_{s}
\end{array}\right] \mathrm{dV}+\int_{\mathrm{V}}\left[\begin{array}{c}
\frac{\partial \mathbf{B}_{m}}{\partial \mathbf{u}_{\mathrm{L}}^{\mathrm{T}}} \\
\mathbf{0}
\end{array}\right]^{\mathrm{T}} \boldsymbol{\sigma} \mathrm{dV}
$$


where $\mathbf{D}_{\mathrm{T}}$ is a symmetric consistent tangent modulus matrix [61,71-72], $\frac{\partial \mathbf{B}_{m}}{\partial \mathbf{u}_{\mathrm{L}}^{\mathrm{T}}}$ is the second derivative of the membrane strain vector $\boldsymbol{\varepsilon}_{m}$ with respect to the local nodal variable vector; the details are given in Appendix A. The first term on the right-hand side of Eq. (20) is symmetric; the second term is also symmetric due to the commutativity in calculating the second derivative of the membrane strain vector $\boldsymbol{\varepsilon}_{m}$ with respect to the local nodal variable vector. Thus the element tangent stiffness matrix $\mathbf{k}_{\mathrm{T}}$ is symmetric.

In the present elasto-plastic shell element, we adopt a condensed elastic constitutive relationship in combination with a yield surface based on the five stress components excluding the normal out-of-plane stress [66,71-72]. This adoption avoids the need for elasto-plastic condensation of the normal out-of-plane stress and achieves the same results with a relatively simple formulation based only on five stress and corresponding strain components. We adopt the Maxwell-Huber-Hencky-von Mises yield criterion together with an isotropic strain hardening. The Maxwell-Huber- Hencky-von Mises yield function is expressed as

$f=\sigma_{e}-\sigma_{y}$

where $\sigma_{e}$ is the equivalent uniaxial stress at the current stress state:

$\sigma_{e}=\sqrt{\sigma_{x x}^{2}+\sigma_{y y}^{2}-\sigma_{x x} \sigma_{y y}+3\left(\tau_{x y}^{2}+\tau_{y z}^{2}+\tau_{z x}^{2}\right)}$

$\boldsymbol{\sigma}^{\mathrm{T}}=\left\lfloor\begin{array}{lllll}\sigma_{x x} & \sigma_{y y} & \tau_{x y} & \tau_{x z} & \tau_{y z}\end{array}\right\rfloor$ represents the current stress state, $\sigma_{y}$ is the current value of uniaxial yield stress, dependent on the accumulated equivalent plastic strain:

$\sigma_{y}=\mathrm{f}_{y}+\mathrm{H} \lambda$

in which $\mathrm{f}_{y}$ is the initial value of uniaxial yield stress, $\mathrm{H}$ is the hardening parameter, and $\lambda$ is the plastic strain multiplier.

In a nonlinear incremental solution procedure, the incremental material strains $\Delta \boldsymbol{\varepsilon}_{j}^{i^{\mathrm{T}}}=\left\lfloor\Delta \varepsilon_{x x{ }_{j}}{ }^{i} \Delta \varepsilon_{y y_{j}}{ }^{i} \Delta \gamma_{x y_{j}}{ }^{i} \Delta \gamma_{x z j}{ }^{i} \Delta \gamma_{y z}{ }^{i}\right\rfloor$, which, under elastic deformation, leads to a stress increment $\Delta \boldsymbol{\sigma}_{j}^{i}$ and an elastic stress state $\boldsymbol{\sigma}_{j}^{i}$. If $f>0$, the stress state $\boldsymbol{\sigma}_{j}^{i}$ falls outside the yield surface, then a plastic material strain increment $\Delta \boldsymbol{\varepsilon}_{p_{j}}{ }^{i}$ must be introduced so that the solution satisfies the flow rule and stays on the yield surface. The backward-Euler return-mapping procedure is then used due to its efficiency and the fact that it results in a symmetric consistent tangent modulus matrix [61]. The details of developing the consistent tangent modulus matrix and the elasto-plastic formulation can be found in [71-72].

\subsection{Strategies for Overcoming Locking Problems}

Eqs. 19 and 20 represent the conforming element formulation for the 3-node triangular shell element in the local coordinate system. In solving thin shell problems, membrane and shear locking phenomena could lead to deterioration in the computational efficiency and accuracy of the 
conforming element. Therefore, to improve the performance of the triangular shell element, the membrane strains and out-of-plane shear strains are replaced with the corresponding assumed strains [42]; accordingly, the modified element formulations are given as follows:

$\mathbf{f}=\int_{\mathrm{V}}\left[\begin{array}{cc}\widetilde{\mathbf{B}}_{m}+z_{l} \mathbf{B}_{b} \\ \\ \widetilde{\mathbf{B}}_{s}\end{array}\right]^{\mathrm{T}} \widetilde{\boldsymbol{\sigma}} \mathrm{dV}$

$\mathbf{k}_{\mathrm{T}}=\int_{\mathrm{V}}\left\{\left[\begin{array}{c}\widetilde{\mathbf{B}}_{m}+z_{l} \mathbf{B}_{b} \\ \widetilde{\mathbf{B}}_{s}\end{array}\right]^{\mathrm{T}} \mathbf{D}_{\mathrm{T}}\left[\begin{array}{c}\widetilde{\mathbf{B}}_{m}+z_{l} \mathbf{B}_{b} \\ \widetilde{\mathbf{B}}_{s}\end{array}\right]+\left[\begin{array}{c}\frac{\partial \widetilde{\mathbf{B}}_{m}}{\partial \mathbf{u}_{\mathrm{L}}^{\mathrm{T}}} \\ \frac{\partial \widetilde{\mathbf{B}}_{s}^{\mathrm{T}}}{\partial \mathbf{u}_{\mathrm{L}}^{\mathrm{T}}}\end{array}\right]^{\mathrm{\sigma}} \widetilde{\boldsymbol{\sigma}}\right\} \mathrm{dV}$

where the matrices $\widetilde{\mathbf{B}}_{m}$ and $\widetilde{\mathbf{B}}_{s}$ are respectively the first derivatives of the assumed membrane strain vector $\widetilde{\boldsymbol{\varepsilon}}_{m}$ and the assumed out-of-plane shear strain vector $\widetilde{\gamma}$ with respect to the local nodal variable vector $\mathbf{u}_{\mathrm{L}}$; the matrices $\frac{\partial \widetilde{\mathbf{B}}_{m}}{\partial \mathbf{u}_{\mathrm{L}}^{\mathrm{T}}}$ and $\frac{\partial \widetilde{\mathbf{B}}_{s}}{\partial \mathbf{u}_{\mathrm{L}}^{\mathrm{T}}}$ are respectively the second derivative of the assumed membrane strain vector $\widetilde{\boldsymbol{\varepsilon}}_{m}$ and the second derivative of the assumed out-of-plane shear strain vector $\widetilde{\gamma}$ with respect to the local nodal variable vector $\mathbf{u}_{\mathrm{L}}$; the details of these matrices are given in Appendix A. The stress vector $\tilde{\boldsymbol{\sigma}}$ is obtained by replacing the incremental material strains $\Delta \boldsymbol{\varepsilon}_{j}^{i}$ with the incremental assumed material strains $\Delta \widetilde{\boldsymbol{\varepsilon}}_{j}^{i}$ [61,71-72], and the incremental assumed material strains $\Delta \widetilde{\boldsymbol{\varepsilon}}_{j}^{i}$ is accumulated from the initial iteration to the $j^{\text {th }}$ iteration of the $i^{\text {th }}$ incremental loading step to avoid the occurrence of "spurious unloading" during the iterations.

The first term in the right-hand side of Eq. (25) is symmetric, and the second term is also symmetric due to the commutativity in calculating the second derivative of the assumed membrane strain vector $\widetilde{\boldsymbol{\varepsilon}}_{m}$ with respect to the local nodal variable vector. Thus the modified element tangent stiffness matrix $\mathbf{k}_{\mathrm{T}}$ is still symmetric.

The assumed material strains are calculated as:

$\widetilde{\boldsymbol{\varepsilon}}=\left\{\begin{array}{c}\widetilde{\boldsymbol{\varepsilon}}_{m}+z_{l} \chi \\ \widetilde{\gamma}\end{array}\right\}$

where the distribution of membrane strains are assumed to be:

$\widetilde{\boldsymbol{\varepsilon}}_{m}=\mathbf{P} \boldsymbol{\alpha}$

$\mathbf{P}=\left[\begin{array}{lll}1 & 0 & 0 \\ 0 & 1 & 0 \\ 0 & 0 & 1\end{array}\right]$

$\boldsymbol{\alpha}^{\mathrm{T}}=\left\lfloor\begin{array}{lll}\alpha_{1} & \alpha_{2} & \alpha_{3}\end{array}\right\rfloor$

and the distribution of the transverse shear strains and the torsion of the top face of the element 
relative to the bottom face (Figure 3 ) are assumed to be:

$$
\begin{aligned}
& \left\{\begin{array}{c}
\widetilde{\gamma} \\
\widetilde{\gamma}_{t}
\end{array}\right\}=\mathbf{P} \boldsymbol{\beta} \\
& \boldsymbol{\beta}^{\mathrm{T}}=\left\lfloor\begin{array}{lll}
\beta_{1} & \beta_{2} & \beta_{3}
\end{array}\right\rfloor
\end{aligned}
$$

with $\alpha_{i}$ and $\beta_{j} \quad(i, j=1,2,3)$ being undetermined assumed strain coefficients.

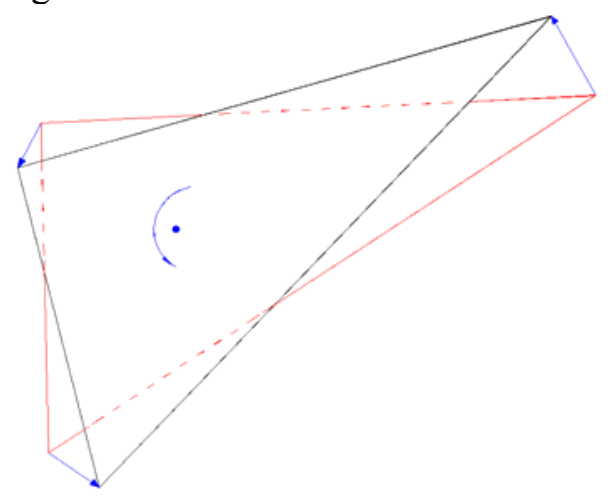

Figure 3. Torsion of the Top Face of the Element Relative to the Bottom Face

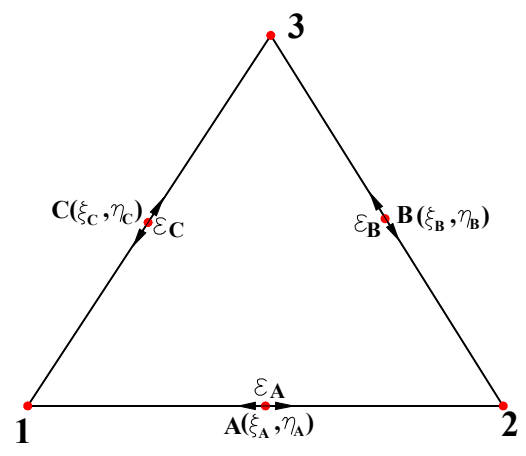

(a)

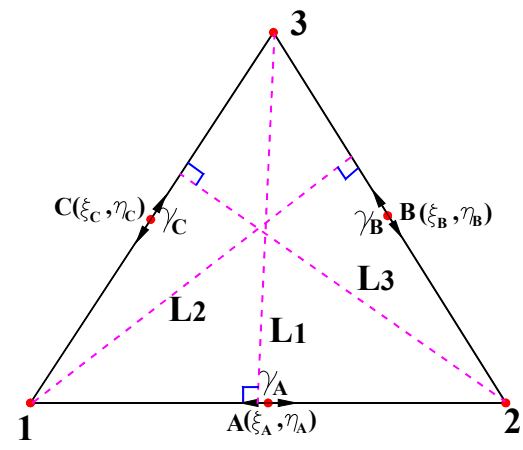

(b)

Figure 4. Member Strains and Edge-member Transverse Shear Strains for Present Shell Element

Inspired by the line integration approach proposed by MacNeal [42], the member strains (namely, the edge-member membrane strains in the direction of each side of the element) corresponding to the membrane strains of the present 3-node triangular shell element are related to the displacements at two neighboring nodes, and are evaluated by

$\varepsilon_{k}=\frac{\left(\mathbf{t}_{j}-\mathbf{t}_{i}\right)^{\mathrm{T}} \mathbf{a}_{i j 0}}{\left|\mathbf{a}_{i j 0}\right|^{2}}+\frac{1}{2}\left(\frac{w_{j}-w_{i}}{\left|\mathbf{a}_{i j 0}\right|}\right)^{2}$

where the ordered triplets $(k, i, j)$ are given by $(\mathrm{A}, 1,2),(\mathrm{B}, 2,3)$ and $(\mathrm{C}, 3,1)$ (see Figure $4 \mathrm{a})$, and

$$
\mathbf{a}_{i j 0}=\mathbf{x}_{j 0}-\mathbf{x}_{i 0}
$$

The relationships of the edge-member strains $\quad \varepsilon_{k}$ and the membrane strains $\quad \boldsymbol{\varepsilon}=\left\lfloor\begin{array}{lll}\varepsilon_{x x} & \varepsilon_{y y} & \gamma_{x y}\end{array}\right\rfloor^{\mathrm{T}}$ at Point $k(k=\mathrm{A}, \mathrm{B}, \mathrm{C})$ are approximately expressed as 
$\varepsilon_{k}=c_{k}^{2} \varepsilon_{x x}+s_{k}^{2} \varepsilon_{y y}+c_{k} s_{k} \gamma_{x y}$

where $c_{k}=\cos \psi_{k}, s_{k}=\sin \psi_{k}$, and $\psi_{k}(k=\mathrm{A}, \mathrm{B}, \mathrm{C})$ is the angle from the $x$-axis to the $i$ - $j$ edge of the element. The natural coordinates $\left(\xi_{k}, \eta_{k}\right)$ of Points $k=\mathrm{A}, \mathrm{B}, \mathrm{C}$ take the values shown in Table 1:

Table 1. The Natural Coordinates of Points A,B and C

\begin{tabular}{cccc}
\hline $\mathrm{k}$ & $\mathrm{A}$ & $\mathrm{B}$ & $\mathrm{C}$ \\
\hline$\xi_{k}$ & 0.5 & 0.5 & 0.0 \\
\hline$\eta_{k}$ & 0.0 & 0.5 & 0.5 \\
\hline
\end{tabular}

Considering Eq. 27, the edge-member strains $\varepsilon_{k}(k=\mathrm{A}, \mathrm{B}, \mathrm{C})$ (see Figure. $4 \mathrm{a}$ ) can be evaluated in terms of the assumed membrane strain coefficients:

$\left\{\varepsilon_{k}\right\}=\boldsymbol{\Gamma} \boldsymbol{\alpha}, \quad k=\mathrm{A}, \mathrm{B}, \mathrm{C}$

$\boldsymbol{\Gamma}=\left[\begin{array}{ccc}c_{\mathrm{A}}^{2} & s_{\mathrm{A}}^{2} & c_{\mathrm{A}} s_{\mathrm{A}} \\ c_{\mathrm{B}}^{2} & s_{\mathrm{B}}^{2} & c_{\mathrm{B}} s_{\mathrm{B}} \\ c_{\mathrm{C}}^{2} & s_{\mathrm{C}}^{2} & c_{\mathrm{C}} s_{\mathrm{C}}\end{array}\right]$

From Eqs. 27 and 35a,b, the assumed membrane strains can be expressed in terms of the edge-member strains $\varepsilon_{k}(k=\mathrm{A}, \mathrm{B}, \mathrm{C})$ as

$\widetilde{\boldsymbol{\varepsilon}}_{m}=\mathbf{P} \Gamma^{-1}\left\{\varepsilon_{k}\right\}, \quad k=\mathrm{A}, \mathrm{B}, \mathrm{C}$

Similarly, the edge-member transverse shear strains $\gamma_{k}(k=\mathrm{A}, \mathrm{B}, \mathrm{C})$ along the 3 edges of the 3 -node triangular shell element (see Figure. $4 \mathrm{~b}$ ) are related to the displacements at two neighboring nodes. Considering that the components of the mid-surface normal vectors $\mathbf{r}$ and $\mathbf{r}_{0}$ along these line segments are linear functions of distance, the edge-member transverse shear strain $\gamma_{k}$ of the edge $i-j$ can be evaluated by

$\gamma_{k}=\frac{w_{j}-w_{i}}{\left|\mathbf{a}_{i j 0}\right|}+\frac{\left(\mathbf{r}_{j}-\mathbf{r}_{j 0}+\mathbf{r}_{i}-\mathbf{r}_{i 0}\right)^{\mathrm{T}} \mathbf{a}_{i j 0}}{2\left|\mathbf{a}_{i j 0}\right|}$

where the ordered triplets $(k, i, j)$ are given by $(\mathrm{A}, 1,2),(\mathrm{B}, 2,3)$ and $(\mathrm{C}, 3,1)$; the first term of Eq. 37 gives the ratio of the incremental out-of-plane displacement from Node $i$ to Node $\mathrm{j}$ with respect to the length of Edge $i j$; the second term is the average of the two projections of the incremental mid-surface normal vectors, at Node $i$ and at Node $j$, on the Edge $i j$.

The edge-member transverse shear strain at Point $k(k=\mathrm{A}, \mathrm{B}, \mathrm{C})$ is calculated from the transverse shear strains and the torsion of the top face of the element relative to the bottom face [42]:

$\gamma_{k}=c_{k} \gamma_{x z}+s_{k} \gamma_{y z}+\gamma_{t}$

Considering Eqs. 30 and 38, the edge-member transverse shear strain $\gamma_{k}$ can be expressed as: 
$\left\{\gamma_{k}\right\}=\mathbf{\Omega} \boldsymbol{\beta}, \quad k=\mathrm{A}, \mathrm{B}, \mathrm{C}$

$\boldsymbol{\Omega}=\left[\begin{array}{lll}c_{\mathrm{A}} & s_{\mathrm{A}} & 1 \\ c_{\mathrm{B}} & s_{\mathrm{B}} & 1 \\ c_{\mathrm{C}} & s_{\mathrm{C}} & 1\end{array}\right]$

According to Eqs. 30 and 39a,b, the assumed shear strains can be given as

$\left\{\begin{array}{c}\tilde{\gamma} \\ \tilde{\gamma}_{t}\end{array}\right\}=\mathbf{P} \boldsymbol{\Omega}^{-1}\left\{\gamma_{k}\right\}, \quad k=\mathrm{A}, \mathrm{B}, \mathrm{C}$

To improve further the accuracy of the present 3-node triangular shell element, the residual bending flexibility is added to the shear flexibility matrix coupling $\tilde{\gamma}_{x z}$ and $\tilde{\gamma}_{y z}$ by dividing the three edge-member transverse shear strains by a correction coefficient, respectively. These coefficients are selected to give correct results for cubic bending in directions perpendicular to the three sides of the triangle [86-87], and are calculated as

$\rho_{k}=\sqrt{1+\frac{5 \mathrm{~L}_{k}^{2}}{24(1+\mu) \mathrm{h}^{2}}}$

where $\mathrm{L}_{k}$ is the height of the triangle corresponding to the edge-member transverse shear strain $\gamma_{k}$ (see Figure $4 \mathrm{~b}$ ), and $\mathrm{h}$ the thickness of the element.

In MacNeal [42], the flexibility assigned to $\gamma_{t}$ was treated as a free parameter to be selected to improve particular test results; the value of this flexibility is unimportant, and is ignored in the presented 3-node triangular shell element.

The incremental form of the assumed material strains is given by

$$
\Delta \widetilde{\boldsymbol{\varepsilon}}=\left\{\begin{array}{c}
\Delta \widetilde{\boldsymbol{\varepsilon}}_{m}+z_{l} \Delta \chi \\
\Delta \widetilde{\gamma}
\end{array}\right\}
$$

in which the vectors $\Delta \widetilde{\boldsymbol{\varepsilon}}_{m}$ and $\Delta \widetilde{\gamma}$ are respectively the incremental form of $\widetilde{\boldsymbol{\varepsilon}}_{m}$ and $\tilde{\gamma}$; these increments can be obtained by the first-order Taylor expansion of Eqs. 36 and 40 with $\Delta \mathbf{t}_{i}, \Delta \mathbf{r}_{i}, \Delta w_{i}$ accumulated from the initial iteration to the current iteration of the present incremental loading step in a nonlinear incremental solution procedure.

\section{TRANSFORMATION OF LOCAL TO GLOBAL RESPONSE}

The global nodal force vector $\mathbf{f}_{g}$ of the triangular shell element can be obtained as a transformation of the local nodal force vector $\mathbf{f}$ according to:

$\mathbf{f}_{g}=\mathbf{T}^{\mathrm{T}} \mathbf{f}$ 
where $\mathbf{T}$ is a $15 \times 15$ transformation matrix consisting of the first derivatives of the local dofs with respect to the global nodal dofs, and can be readily determined from Eqs. 11a,c.

For convenience, the local nodal dofs and the global nodal dofs are rewritten below

$$
\begin{aligned}
& \mathbf{u}_{\mathrm{L}}^{\mathrm{T}}=\left[\begin{array}{llllll}
\mathbf{t}_{1}^{\mathrm{T}} & \boldsymbol{\theta}_{1}^{\mathrm{T}} & \mathbf{t}_{2}^{\mathrm{T}} & \boldsymbol{\theta}_{2}^{\mathrm{T}} & \mathbf{t}_{3}^{\mathrm{T}} & \boldsymbol{\theta}_{3}^{\mathrm{T}}
\end{array}\right] \\
& \mathbf{u}_{\mathrm{G}}^{\mathrm{T}}=\left[\begin{array}{llllll}
\mathbf{d}_{1}^{\mathrm{T}} & \mathbf{n}_{\mathrm{g} 1}^{\mathrm{T}} & \mathbf{d}_{2}^{\mathrm{T}} & \mathbf{n}_{\mathrm{g} 2}^{\mathrm{T}} & \mathbf{d}_{3}^{\mathrm{T}} & \mathbf{n}_{\mathrm{g} 3}^{\mathrm{T}}
\end{array}\right]
\end{aligned}
$$

where $\boldsymbol{\theta}_{k}^{\mathrm{T}}=\left\lfloor r_{k, x} \quad r_{k, y}\right\rfloor$ represents the two local vectorial rotational dofs at Node $k$ in the local

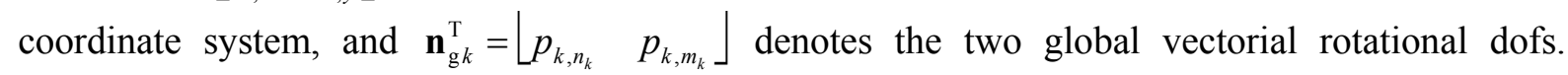
Accordingly, the transformation matrix is given as follows:

$$
\mathbf{T}=\frac{\partial \mathbf{u}_{\mathrm{L}}}{\partial \mathbf{u}_{\mathrm{G}}^{\mathrm{T}}}=\left[\begin{array}{cccccc}
\frac{\partial \mathbf{t}_{1}}{\partial \mathbf{d}_{1}^{\mathrm{T}}} & \mathbf{0} & \frac{\partial \mathbf{t}_{1}}{\partial \mathbf{d}_{2}^{\mathrm{T}}} & \mathbf{0} & \frac{\partial \mathbf{t}_{1}}{\partial \mathbf{d}_{3}^{\mathrm{T}}} & \mathbf{0} \\
\frac{\partial \boldsymbol{\theta}_{1}}{\partial \mathbf{d}_{1}^{\mathrm{T}}} & \frac{\partial \boldsymbol{\theta}_{1}}{\partial \mathbf{n}_{\mathrm{g} 1}^{\mathrm{T}}} & \frac{\partial \boldsymbol{\theta}_{1}}{\partial \mathbf{d}_{2}^{\mathrm{T}}} & \frac{\partial \boldsymbol{\theta}_{1}}{\partial \mathbf{n}_{\mathrm{g} 2}^{\mathrm{T}}} & \frac{\partial \boldsymbol{\theta}_{1}}{\partial \mathbf{d}_{3}^{\mathrm{T}}} & \frac{\partial \boldsymbol{\theta}_{1}}{\partial \mathbf{n}_{\mathrm{g} 3}^{\mathrm{T}}} \\
\frac{\partial \mathbf{t}_{2}}{\partial \mathbf{d}_{1}^{\mathrm{T}}} & \mathbf{0} & \frac{\partial \mathbf{t}_{2}}{\partial \mathbf{d}_{2}^{\mathrm{T}}} & \mathbf{0} & \frac{\partial \mathbf{t}_{2}}{\partial \mathbf{d}_{3}^{\mathrm{T}}} & \mathbf{0} \\
\frac{\partial \boldsymbol{\theta}_{2}}{\partial \mathbf{d}_{1}^{\mathrm{T}}} & \frac{\partial \boldsymbol{\theta}_{2}}{\partial \mathbf{n}_{\mathrm{g} 1}^{\mathrm{T}}} & \frac{\partial \boldsymbol{\theta}_{2}}{\partial \mathbf{d}_{2}^{\mathrm{T}}} & \frac{\partial \boldsymbol{\theta}_{2}}{\partial \mathbf{n}_{\mathrm{g} 2}} & \frac{\partial \boldsymbol{\theta}_{2}}{\partial \mathbf{d}_{3}^{\mathrm{T}}} & \frac{\partial \boldsymbol{\theta}_{2}}{\partial \mathbf{n}_{\mathrm{g} 3}^{\mathrm{T}}} \\
\frac{\partial \mathbf{t}_{3}}{\partial \mathbf{d}_{1}^{\mathrm{T}}} & \mathbf{0} & \frac{\partial \mathbf{t}_{3}}{\partial \mathbf{d}_{2}^{\mathrm{T}}} & \mathbf{0} & \frac{\partial \mathbf{t}_{3}}{\partial \mathbf{d}_{3}^{\mathrm{T}}} & \mathbf{0} \\
\frac{\partial \boldsymbol{\theta}_{3}}{\partial \mathbf{d}_{1}^{\mathrm{T}}} & \frac{\partial \boldsymbol{\theta}_{3}}{\partial \mathbf{n}_{\mathrm{g} 1}^{\mathrm{T}}} & \frac{\partial \boldsymbol{\theta}_{3}}{\partial \mathbf{d}_{2}^{\mathrm{T}}} & \frac{\partial \boldsymbol{\theta}_{3}}{\partial \mathbf{n}_{\mathrm{g} 2}^{\mathrm{T}}} & \frac{\partial \boldsymbol{\theta}_{3}}{\partial \mathbf{d}_{3}^{\mathrm{T}}} & \frac{\partial \boldsymbol{\theta}_{3}}{\partial \mathbf{n}_{\mathrm{g} 3}^{\mathrm{T}}}
\end{array}\right]
$$

with the details of the sub-matrices of $\mathbf{T}$ presented in Appendix B.

The element tangent stiffness matrix $\mathbf{k}_{\mathrm{TG}}$ in the global coordinate system can now be obtained as follows:

$$
\begin{aligned}
& \mathbf{k}_{\mathrm{TG}}=\frac{\partial \mathbf{f}_{\mathrm{g}}}{\partial \mathbf{u}_{\mathrm{G}}^{\mathrm{T}}}=\mathbf{T}^{\mathrm{T}} \frac{\partial \mathbf{f}}{\partial \mathbf{u}_{\mathrm{G}}^{\mathrm{T}}}+\frac{\partial \mathbf{T}^{\mathrm{T}}}{\partial \mathbf{u}_{\mathrm{G}}^{\mathrm{T}}} \mathbf{f}=\mathbf{T}^{\mathrm{T}} \frac{\partial \mathbf{f}}{\partial \mathbf{u}_{\mathrm{L}}^{\mathrm{T}}} \mathbf{T}+\frac{\partial \mathbf{T}^{\mathrm{T}}}{\partial \mathbf{u}_{\mathrm{G}}^{\mathrm{T}}} \mathbf{f} \Rightarrow \\
& \mathbf{k}_{\mathrm{TG}}=\mathbf{T}^{\mathrm{T}} \mathbf{k}_{\mathrm{T}} \mathbf{T}+\frac{\partial \mathbf{T}^{\mathrm{T}}}{\partial \mathbf{u}_{\mathrm{G}}^{\mathrm{T}}} \mathbf{f}
\end{aligned}
$$

with: 


$$
\frac{\partial \mathbf{T}}{\partial \mathbf{u}_{\mathrm{G}}^{\mathrm{T}}}=\left[\begin{array}{ccccc}
\frac{\partial^{2} \mathbf{t}_{1}}{\partial \mathbf{d}_{1}^{\mathrm{T}} \partial \mathbf{u}_{\mathrm{G}}^{\mathrm{T}}} & \mathbf{0} & \cdots & \frac{\partial^{2} \mathbf{t}_{1}}{\partial \mathbf{d}_{3}^{\mathrm{T}} \partial \mathbf{u}_{\mathrm{G}}^{\mathrm{T}}} & \mathbf{0} \\
\frac{\partial^{2} \boldsymbol{\theta}_{1}}{\partial \mathbf{d}_{1}^{\mathrm{T}} \partial \mathbf{u}_{\mathrm{G}}^{\mathrm{T}}} & \frac{\partial^{2} \boldsymbol{\theta}_{1}}{\partial \mathbf{n}_{\mathrm{g} 1}^{\mathrm{T}} \partial \mathbf{u}_{\mathrm{G}}^{\mathrm{T}}} & \cdots & \frac{\partial^{2} \boldsymbol{\theta}_{1}}{\partial \mathbf{d}_{3}^{\mathrm{T}} \partial \mathbf{u}_{\mathrm{G}}^{\mathrm{T}}} & \frac{\partial^{2} \boldsymbol{\theta}_{1}}{\partial \mathbf{n}_{\mathrm{g} 3}^{\mathrm{T}} \partial \mathbf{u}_{\mathrm{G}}^{\mathrm{T}}} \\
\vdots & \vdots & \vdots & \vdots & \vdots \\
\frac{\partial^{2} \mathbf{t}_{3}}{\partial \mathbf{d}_{1}^{\mathrm{T}} \partial \mathbf{u}_{\mathrm{G}}^{\mathrm{T}}} & \mathbf{0} & \cdots & \frac{\partial^{2} \mathbf{t}_{3}}{\partial \mathbf{d}_{3}^{\mathrm{T}} \partial \mathbf{u}_{\mathrm{G}}^{\mathrm{T}}} & \mathbf{0} \\
\frac{\partial^{2} \boldsymbol{\theta}_{3}}{\partial \mathbf{d}_{1}^{\mathrm{T}} \partial \mathbf{u}_{\mathrm{G}}^{\mathrm{T}}} & \frac{\partial^{2} \boldsymbol{\theta}_{3}}{\partial \mathbf{n}_{\mathrm{g} 1}^{\mathrm{T}} \partial \mathbf{u}_{\mathrm{G}}^{\mathrm{T}}} & \cdots & \frac{\partial^{2} \boldsymbol{\theta}_{3}}{\partial \mathbf{d}_{3}^{\mathrm{T}} \partial \mathbf{u}_{\mathrm{G}}^{\mathrm{T}}} \frac{\partial^{2} \boldsymbol{\theta}_{3}}{\partial \mathbf{n}_{\mathrm{g} 3}^{\mathrm{T}} \partial \mathbf{u}_{\mathrm{G}}^{\mathrm{T}}}
\end{array}\right]
$$

where the various second derivatives in Eq. 48 are given in Appendix B. In the right side of Eq. 47, the first term is symmetric. Considering the commutativity of the global nodal variables in the differentiation of Eq. 48, the second term (its component at $j^{\text {th }}$ row and $k^{\text {th }}$ column is $\sum_{i=1}^{15} \frac{\partial u_{\mathrm{L} i}}{\partial u_{\mathrm{G} j} \partial u_{\mathrm{G} k}} f_{i}, j, k=1,2, \ldots, 15 ; u_{\mathrm{L} i}$ and $f_{i}$ are respectively the $i^{\text {th }}$ components of $\mathbf{u}_{\mathrm{L}}$ and $\mathbf{f}$; $u_{\mathrm{G} j}$ and $u_{\mathrm{G} k}$ are respectively the $j^{\text {th }}$ and $k^{\text {th }}$ components of $\mathbf{u}_{\mathrm{G}}$ ) in the right side of Eq. 47 is also symmetric, so the element tangent stiffness matrix in the global coordinate system is symmetric.

\section{NUMERICAL EXAMPLES}

In the present newly developed 3-node co-rotational triangular shell element (abbreviated to "TRIS3" element in the following examples), assumed membrane strains and shear strains are employed to alleviate membrane and shear locking problems. To demonstrate the reliability and convergence of the TRIS3 element in solving elastic and elasto-plastic plate/shell problems with large displacements, (1) two plate patch tests, (2) three elastic plate/shell problems, and (3) three elasto-plastic plate/shell problems are analyzed using this element. With regard to numerical integration over the thickness, 2 Gauss points are adopted for the two patch tests (Example 5.1) and the three elastic plate/shell problems (Examples 5.2-5.4), and 6 Gauss points are adopted for the three elasto-plastic plate/shell problems (Examples 5.5-5.7) in accordance with the corresponding references considering these examples. Meanwhile, one Gauss point is adopted in numerical integration over the element domain for all examples. The results are also compared to those from other researchers $[36,73-81]$.

\subsection{Patch Tests}

Two patch tests for the membrane behavior and the transverse out-of-plane bending behavior of plate and shell elements were suggested by MacNeal and Harder [73]. The linear results from the TRIS3 element agree exactly with the theoretical results [73].

\subsection{Clamped Annular Plate Strip subject to Transverse Uniformly-Distributed End Load}

An annular plate strip has a geometry of the internal radius $\mathrm{r}=6 \mathrm{~m}$, the external radius $\mathrm{R}=10 \mathrm{~m}$, and the thickness $\mathrm{h}=0.03 \mathrm{~m}$. Its elastic modulus and Poisson's ratio are $\mathrm{E}=2.1 \times 10^{8} \mathrm{KN} / \mathrm{m}^{2}$ and $\mu=0.0$, respectively. The plate is laid horizontally, and clamped at one end and uniformly loaded on the other end. The load is in downward direction along the free edge (see Figure 5), $q=6 \mathrm{KN} / \mathrm{m}$. 


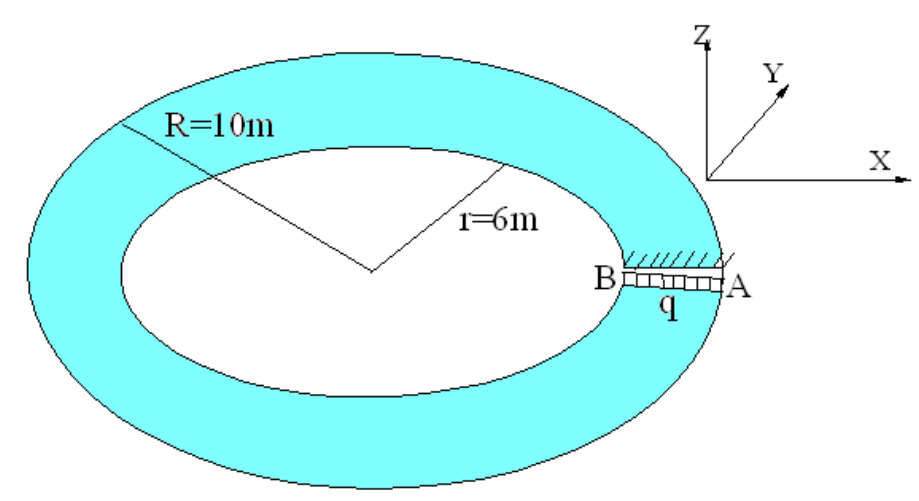

Figure 5. Clamped Annular Plate under Uniformly Distributed Line Load

The load-deflection curves at Points A and B of the plate calculated respectively by using a $4 \times 32 \times 2$ TRIS3-element mesh ( the $1^{\text {st }}$ two numbers ' $4 \times 32$ ' represents a mesh of quadrilateral elements, with the $3^{\text {rd }}$ number ' $\times 2$ ' indicating that each quadrilateral element is further subdivided into 2 triangular elements), a $8 \times 64 \times 2$ TRIS3-element mesh, and a $16 \times 128 \times 2$ TRIS3-element mesh are presented in Figure 6 , where it can be observed that the results obtained with the $4 \times 32 \times 2$ TRIS3-element mesh are already accurate compared with the even more accurate results using the $8 \times 64 \times 2$ TRIS3-element mesh or the $16 \times 128 \times 2$ TRIS3-element mesh. For comparison purpose, the results from Campello et al.[36] and Buechter \& Ramm [74] are also depicted in this figure. The solutions obtained from using $4 \times 32 \times 2,8 \times 64 \times 2$ and $16 \times 128 \times 2$ lower order TRIS3 elements compare favourably with those from Campello et al.[36], employing $8 \times 64$ higher order six-node triangular shell elements, and Buechter \& Ramm [74], employing $2 \times 16$ higher order bi-cubic quadrilateral shell elements.

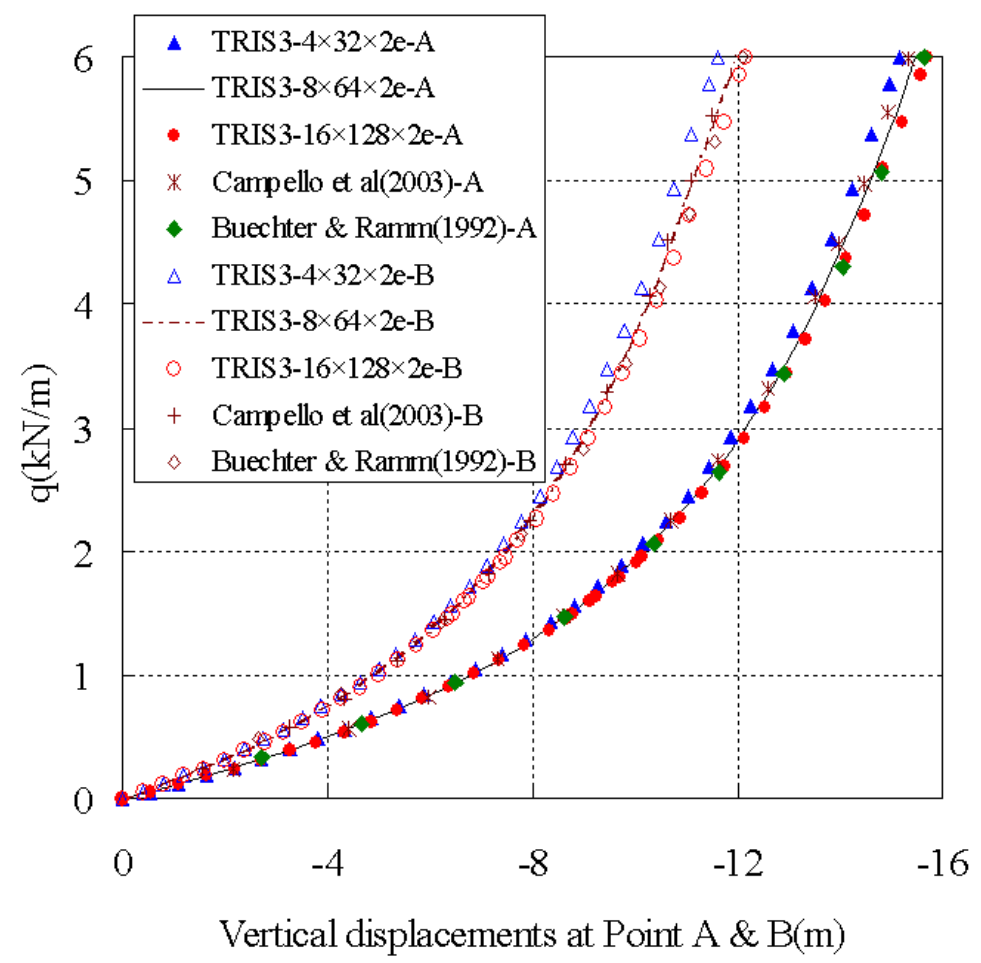

Figure 6. Load-deflection Curves at Points A and B of the Clamped Annular Plate 
Table 2. Deflections at Points A and B of the Clamped Annular Plate under End Load q=6KN/m

\begin{tabular}{ccccc}
\hline Element mesh & \multicolumn{2}{c}{ Deflection at Point A } & \multicolumn{2}{c}{ Deflection at Point B } \\
\hline TRIS3-4 $\times 32 \times 2$ & $-15.1581 \mathrm{~m}$ & $-3.48 \%$ & $-11.6028 \mathrm{~m}$ & $-4.67 \%$ \\
\hline TRIS3-8 $\times 64 \times 2$ & $-15.4847 \mathrm{~m}$ & $-1.40 \%$ & $-11.9445 \mathrm{~m}$ & $-1.86 \%$ \\
\hline TRIS3-16 $\times 128 \times 2$ & $-15.7051 \mathrm{~m}$ & -- & $-12.1711 \mathrm{~m}$ & -- \\
\hline
\end{tabular}

To evaluate the convergence of the solutions obtained with the TRIS3 element using respectively the $4 \times 32 \times 2,8 \times 64 \times 2$ and $16 \times 128 \times 2$ meshes, the deflections at Points A and B of the clamped annular plate under end loading $\mathrm{q}=6 \mathrm{KN} / \mathrm{m}$ and their relative errors based on the results from the highest-density $16 \times 128 \times 2$ TRIS3-element mesh are presented in Table 2 .

The deformed shape of the clamped annular plate under end load $\mathrm{q}=6 \mathrm{KN} / \mathrm{m}$ obtained using $4 \times 32 \times 2$ TRIS3 elements is presented in Figure 7, where large displacements and rotations are evident.

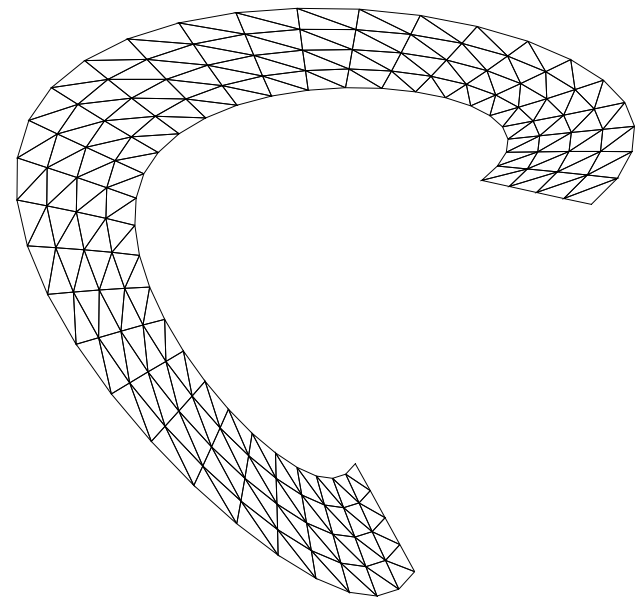

Figure 7. Deformed Shape of the Clamped Annular Plate under End Load q=6KN $/ \mathrm{m}$

\subsection{Lateral Buckling of L-shaped Plate Strip}

A flat L-shaped plate strip is fully clamped on one edge and subjected to an in-plane point load at the free end (Figure 8), having Young's modulus E $=71,240 \mathrm{~N} / \mathrm{mm}^{2}$, and Poisson's ratio $\mu=0.31$. The plate has a geometry of $\mathrm{L}=240 \mathrm{~mm}, \mathrm{~b}=30 \mathrm{~mm}$, and $\mathrm{h}=0.6 \mathrm{~mm}$.

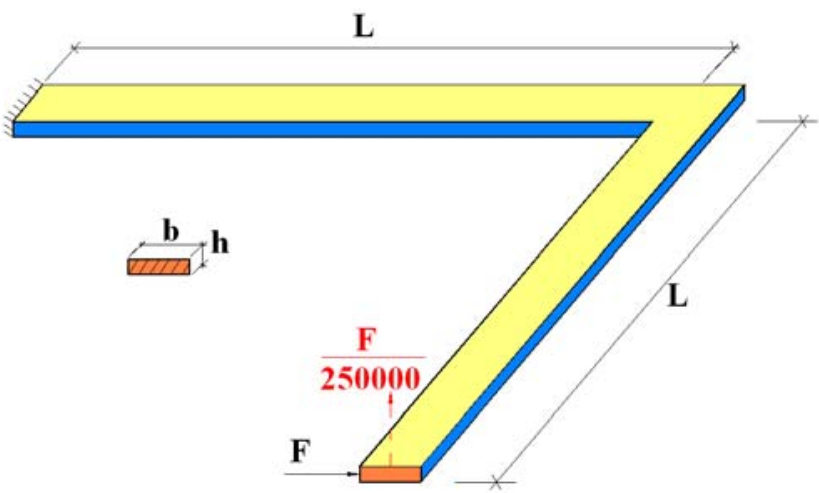

Figure 8. Fully Clamped L-shaped Plate Strip subject to in-plane Point Load at Free End

To investigate the lateral stability of the L-shaped plate, a very small perturbation load $(\mathrm{F} / 250,000)$ 
is imposed on the free edge in the out-of-plane direction to trigger the post-critical lateral deflection (see Figure 8). The meshes used for this analysis are a $64 \times 2$ TRIS3-element mesh (where, ' 64 ' represents quadrilateral elements, ' $\times 2$ ' means that each quadrilateral element is further subdivided into 2 triangular elements), a $256 \times 2$ TRIS3-element mesh, and a $1024 \times 2$ TRIS3-element mesh; the results are presented in Figure 9. To verify the reliability of the present TRIS3 element, the results from a mesh with 136 six-node triangular shell elements [36] and a mesh with 68 four-node EAS elements [75] are also represented in Figure 9.

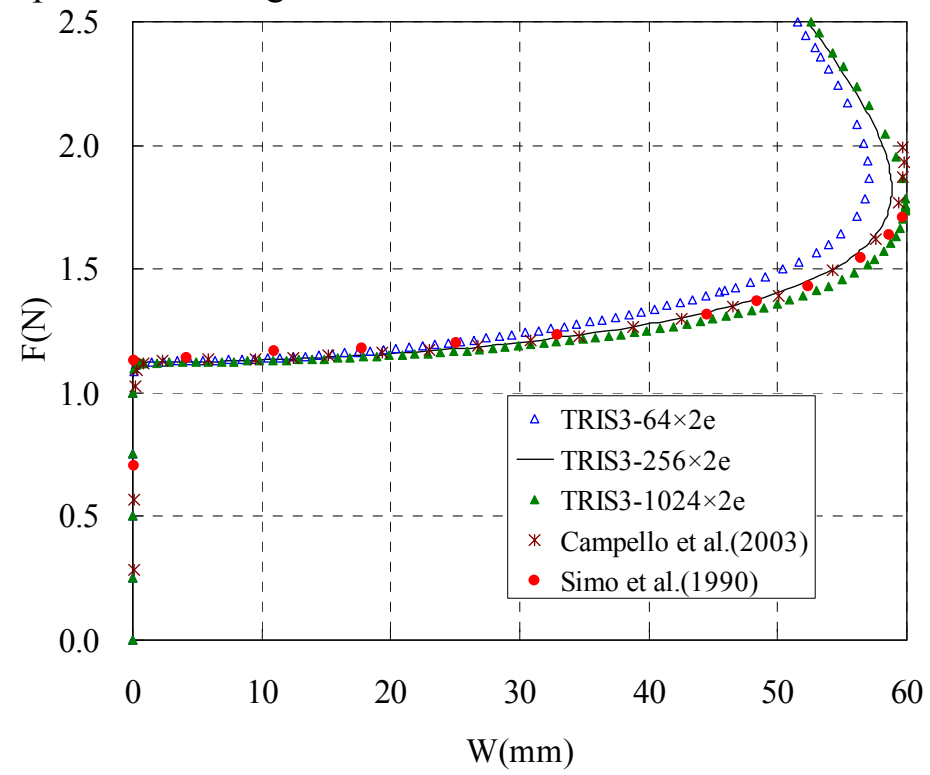

Figure 9. Out-of-plane Lateral Deflection at Free End of L-shaped Plate

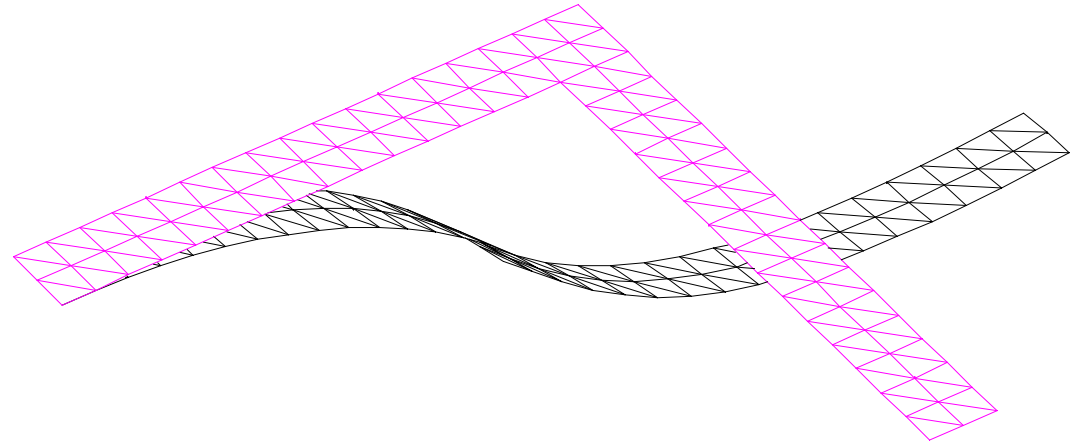

Figure 10. L-shaped Plate: Initial and Deformed Shape

The deformed shape of the L-shape plate (with the $64 \times 2$ TRIS3-element mesh) at the load level of $\mathrm{F}=2.5 \mathrm{~N}$ is presented in Figure 10, where large displacement and large rotation can be observed in the post-buckling stage of the L-shape plate.

\subsection{Pull-out of Open Cylindrical Shell}

An open cylindrical shell has a geometry of $\mathrm{L}=10.35, \mathrm{R}=4.953$, and $\mathrm{h}=0.094$. Its material properties are $\mathrm{E}=10.5 \times 10^{6}$ and $\mu=0.3125$, respectively. The cylindrical shell is pulled by two diametrically opposite point forces F (Figure 11). For symmetry reasons, only one-eighth of the cylindrical shell (i.e. the colored part) is analyzed. 


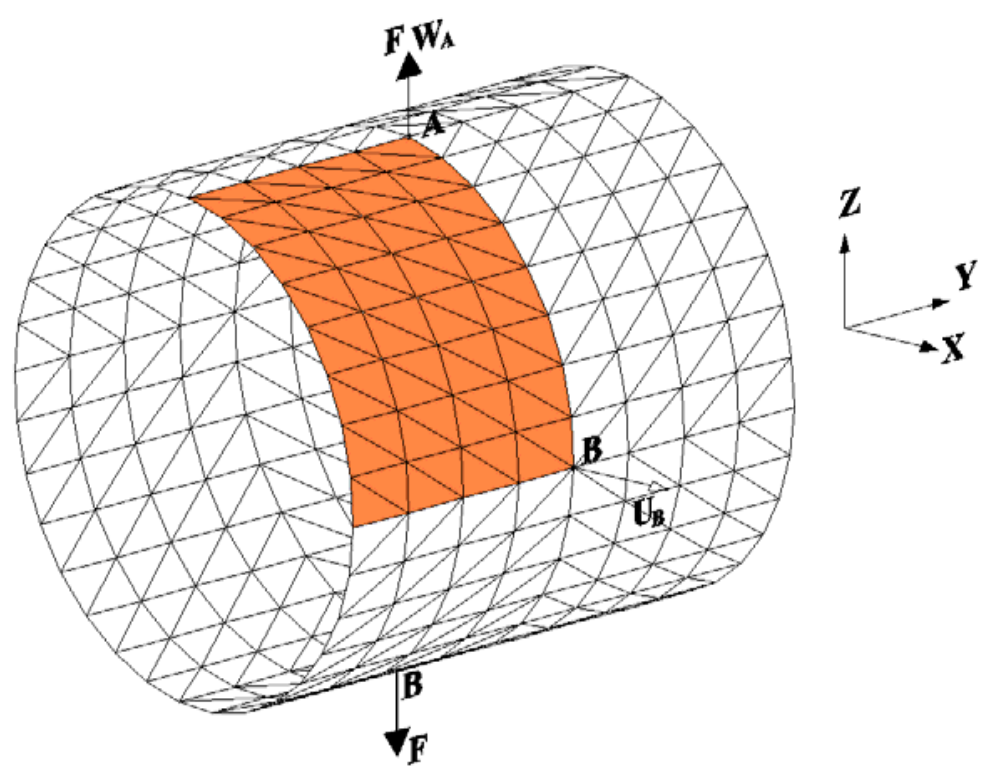

Figure 11. Open Cylindrical Shell subject to Two Diametrically Opposite Point Forces

Three meshes with $4 \times 8 \times 2,8 \times 16 \times 2$ and $16 \times 32 \times 2$ TRIS3 elements are employed respectively to calculate the deflection at the loading point $\mathrm{A}$ of the cylindrical shell. For comparison, we also represent in Figure12 the results from Campello et al. [36] using a $8 \times 16 \times 2$ mesh of six-node triangular elements, the results from Jiang \& Chernuka [76] using a $8 \times 12$ mesh of four-node ANS degenerated-shell elements, and the results from Sze et al. [77] using a $8 \times 12$ mesh of eight-node hybrid-stress solid-shell element based on the total Lagrangian framework.

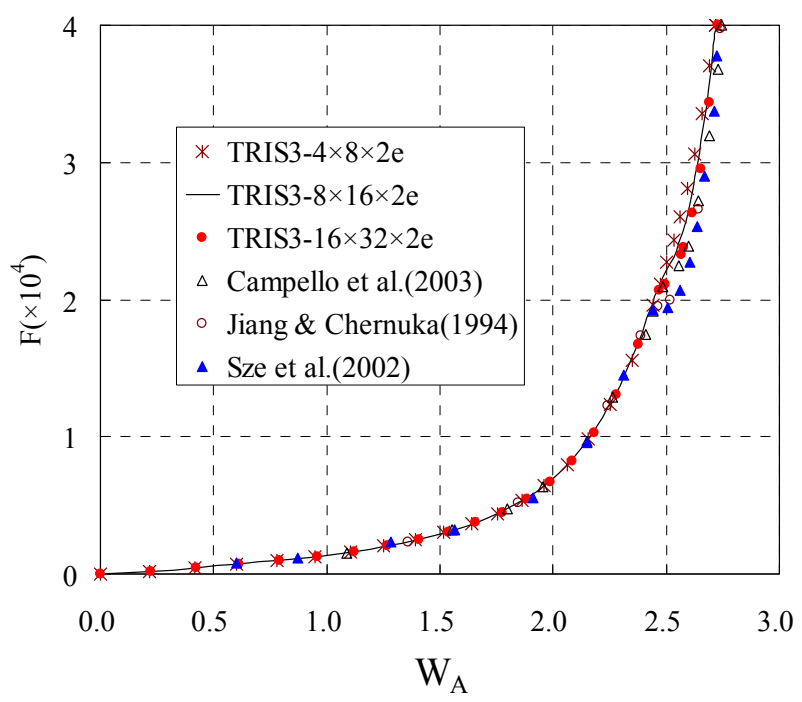

Figure 12. Load-deflection Curve at Point A of Open Cylindrical Shell

To demonstrate the convergence of the present element, the solution calculated respectively from using the meshes with $4 \times 8 \times 2,8 \times 18 \times 2,16 \times 32 \times 2$ and $32 \times 64 \times 2$ TRIS 3 elements are presented in Table 3 , with the relative errors based on the results from the highest-density $32 \times 64 \times 2$ mesh. 


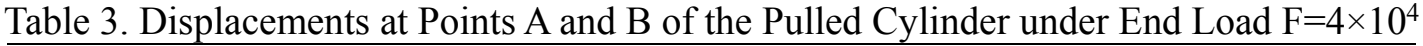

\begin{tabular}{ccccc}
\hline Element meshes & \multicolumn{2}{c}{ W A (Displacement at Point A) } & \multicolumn{2}{c}{ UB (Displacement at Point B) } \\
\hline TRIS3-4 $\times 8 \times 2$ & 2.7169 & $0.90 \%$ & -4.5083 & $-0.94 \%$ \\
\hline TRIS3-8 $\times 16 \times 2$ & 2.7207 & $0.76 \%$ & -4.5606 & $-0.20 \%$ \\
\hline TRIS3-16 $\times 32 \times 2$ & 2.7295 & $0.44 \%$ & -4.5553 & $-0.08 \%$ \\
\hline TRIS3-32 $\times 64 \times 2$ & 2.7416 & -- & -4.5515 & -- \\
\hline
\end{tabular}

The deformed shape of the pulled cylindrical shell at the load level of $F=40,000$ is presented in Figure 13.

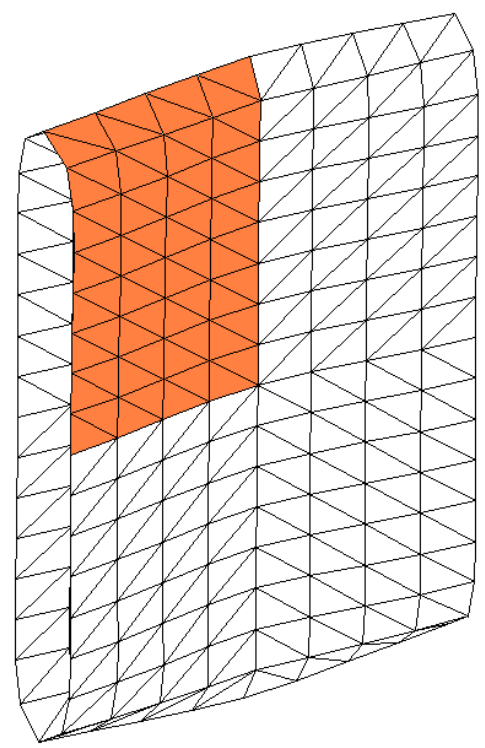

Figure 13. Deformed Shape of the Open Cylindrical Shell

\subsection{Square Plate subjected to Constant Pressure Load}

A square plate is simply supported along the edges (the out-of-plane displacements along its four edges are restrained), and subjected to a deformation dependent pressure load $\mathrm{q}=\mathrm{f} \times \mathrm{p}_{0}$ on one side (Figure 14), where $\mathrm{p}_{0}=10^{-2} \mathrm{~N} / \mathrm{mm}^{2}$. The plate has side length of $2 \mathrm{~L}=508 \mathrm{~mm}$ and thickness $\mathrm{h}=2.54$ $\mathrm{mm}$, with material properties: Young's modulus $\mathrm{E}=6.9 \times 10^{4} \mathrm{~N} / \mathrm{mm}^{2}$, Poisson's ratio $\mu=0.3$, initial yield stress $\mathrm{f}_{\mathrm{y}}=248 \mathrm{~N} / \mathrm{mm}^{2}$, and hardening parameter $\mathrm{H}=0.0$.

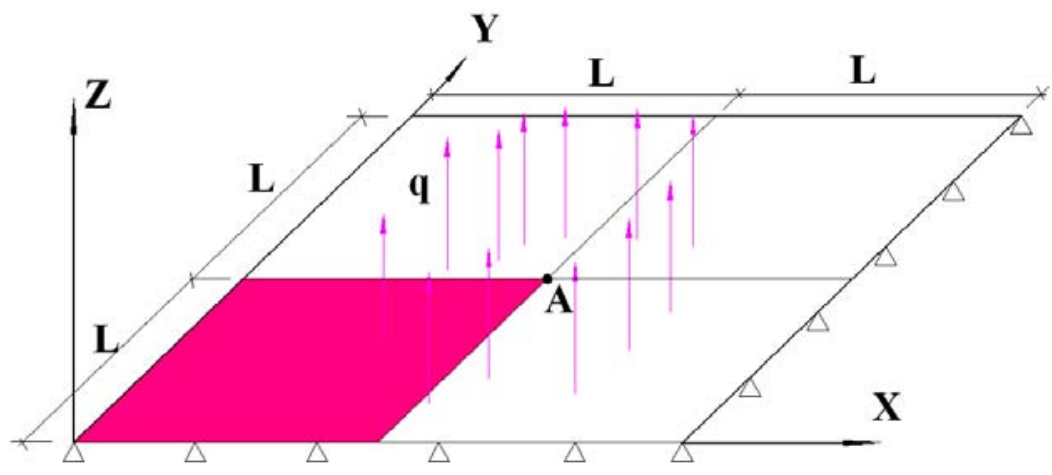

Figure 14. A Simply-supported Square Plate 
Due to symmetry, only a quarter of the plate (the colored zone in Figure 14) is analyzed using respectively a mesh of $12 \times 12 \times 2$, a mesh of $24 \times 24 \times 2$, and a mesh of $48 \times 48 \times 2$ TRIS 3 elements. The load-deflection curves at the central point of the plate, from our present element, are presented in Figure 15. For comparison, the following results are also presented in this figure: (1) Eberlein \& Wriggers [78] using $15 \times 15$ five- or six-parameter quadrilateral 4-node elements with refined mesh toward the outer corner, (2) Betsch \& Stein [79] using $24 \times 24$ quadrilateral 4-node shell elements with regular mesh, (3) Valente et al.[80] using more refined mesh of 1375 S4E6P5 elements and $24 \times 24$ S4E6P5 elements with regular mesh. The results from the three meshes with $12 \times 12 \times 2$, $24 \times 24 \times 2$, and $48 \times 48 \times 2$ of the present TRIS3 elements agree well with those from Betsch \& Stein [79] and Valente et al.[80].

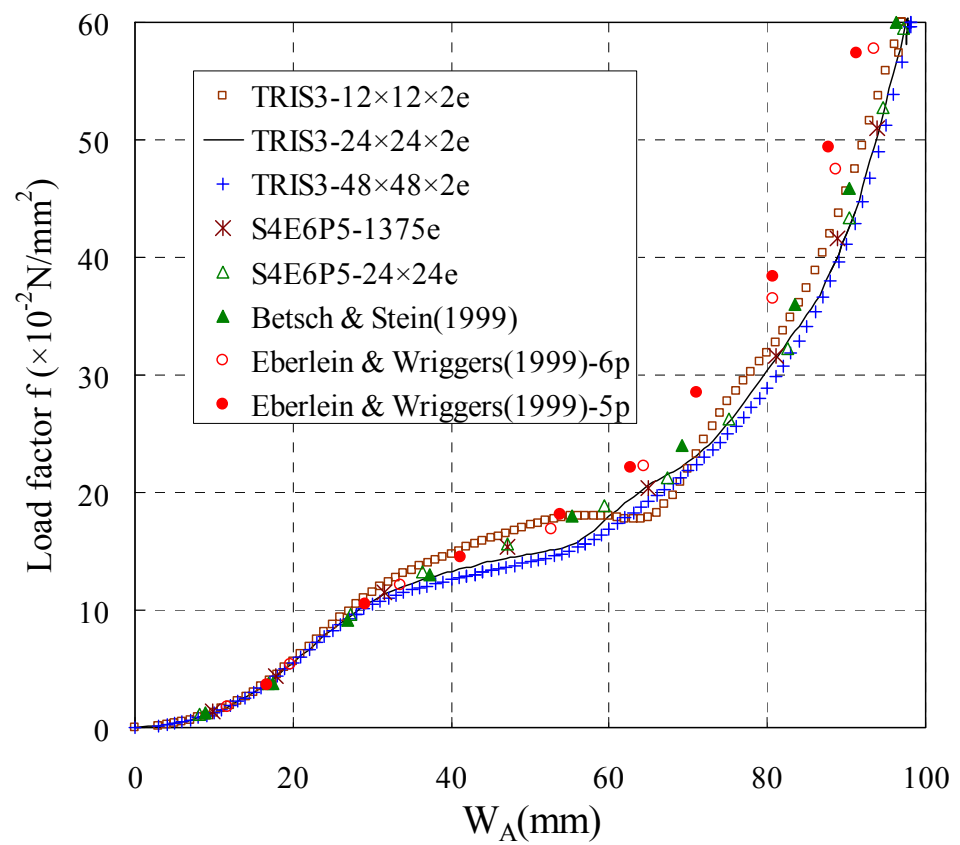

Figure 15. Load-deflection Curves at the Central Point of the Plate

Table 4. Deflection at the Central Point of the Plate under $\mathrm{q}=0.6 \mathrm{~N} / \mathrm{mm}^{2}$

\begin{tabular}{ccc}
\hline Element meshes & \multicolumn{2}{c}{ Deflection at Point A } \\
\hline TRIS3-12 $\times 12 \times 2$ & $96.9707 \mathrm{~mm}$ & $-1.19 \%$ \\
\hline TRIS3-24 $\times 24 \times 2$ & $97.6775 \mathrm{~mm}$ & $-0.47 \%$ \\
\hline TRIS3-48 $\times 48 \times 2$ & $98.1339 \mathrm{~mm}$ & -- \\
\hline
\end{tabular}

To evaluate the convergence of the TRIS3 element, the deflections at the central point of the plate under the pressure of $0.6 \mathrm{~N} / \mathrm{mm}^{2}$ calculated from using the meshes with $12 \times 12 \times 2,24 \times 24 \times 2$ and $48 \times 48 \times 2$ TRIS 3 elements are presented in Table 4 .

In our analysis, the deformation-dependent pressure load is first decomposed in the directions of the three global coordinate axes by multiplying the value of the pressure with the mid-surface normal vector at each integration point on the mid-surface of the plate, the loads at the integration points are then transformed into equivalent nodal loads. The mid-surface normal vectors at integration points are updated at each iteration of every incremental step. We omit the geometric stiffness matrix corresponding to a pressure load to avoid the occurrence of an asymmetric element tangent stiffness matrix within the co-rotational framework. We note, however, that the convergence of the iterative solution procedure in the present problem is not compromised by such omission. 
The deformed shape of the plate at the load level of $\mathrm{q}=0.6 \mathrm{~N} / \mathrm{mm}^{2}$ is presented in Figure 16, showing large displacement and large rotation; plastic zone occurred in the plate.

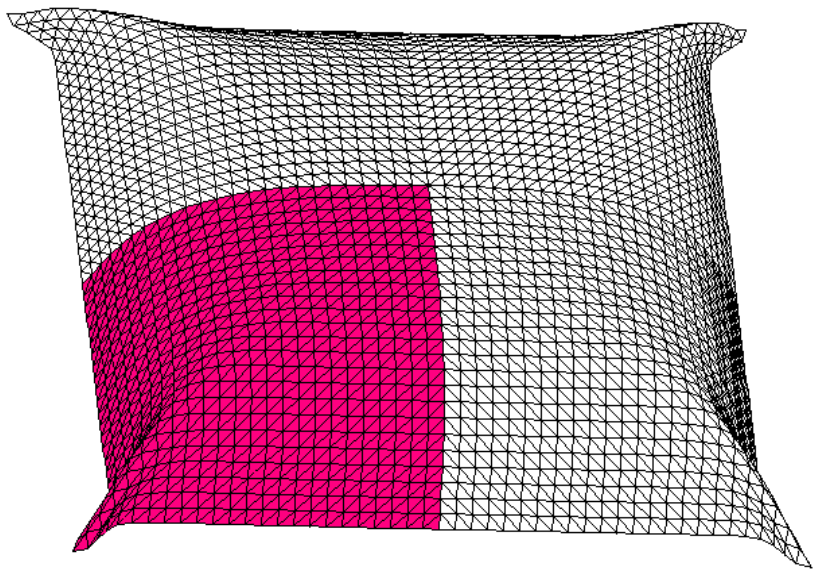

Figure 16. Deformed Shape of Plate under Constant Pressure of $0.60 \mathrm{~N} / \mathrm{mm}^{2}$

\subsection{Pinched Hemispherical Shell}

A hemispherical shell is loaded by two inward and two outward forces at the quarter points of its open edge (Figure 17); the shell has a radius of 10 and a thickness of 0.5 . The material parameters are $\mathrm{E}=10.0, \mu=0.2, \mathrm{f}_{\mathrm{y}}=0.2$ and $\mathrm{H}=9.0$, respectively.

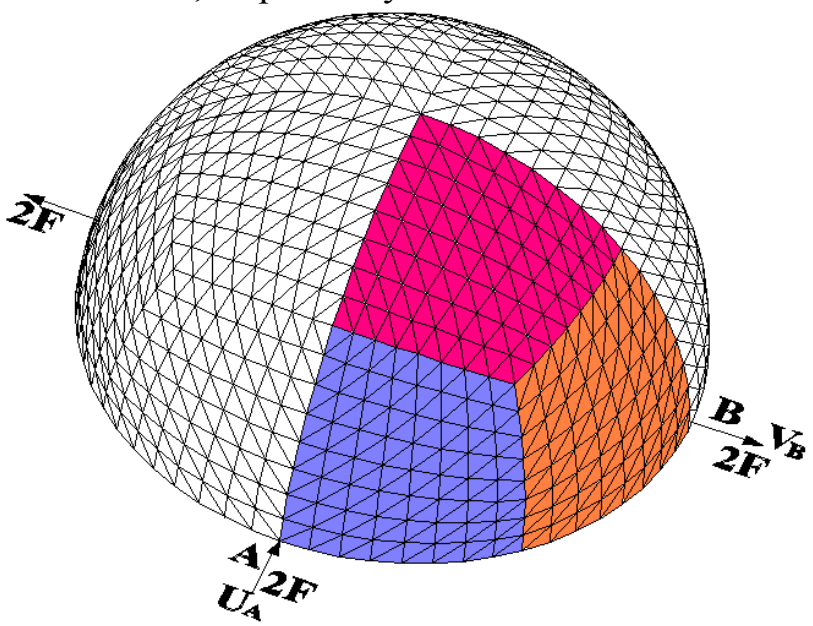

Figure 17. Hemispherical Shell subject to Pinched Forces

Due to symmetry, only a quarter of this hemispherical shell is analyzed (see the colored zone of Figure 17), using a mesh composed of three subdomains, with each subdomain discretized with either (a) $4 \times 4 \times 2$, or (b) $8 \times 8 \times 2$, or (c) $16 \times 16 \times 2$ TRIS3 elements. The load-displacement curves at the pinching points $\mathrm{A}$ and $\mathrm{B}$ are depicted in Figure 18, where it can be observed that the results obtained with three subdomains of $8 \times 8 \times 2$ TRIS 3 elements are already accurate compared with the even more accurate results using three subdomains of $16 \times 16 \times 2$ TRIS3 elements. These results also agree well with those from Bestch \& Stein [79] using three subdomains of $16 \times 16$ bi-linear quadrilateral shell elements, and Eberlein \& Wriggers [78] using three subdomains of $12 \times 12$ quadrilateral 4-node elements. 


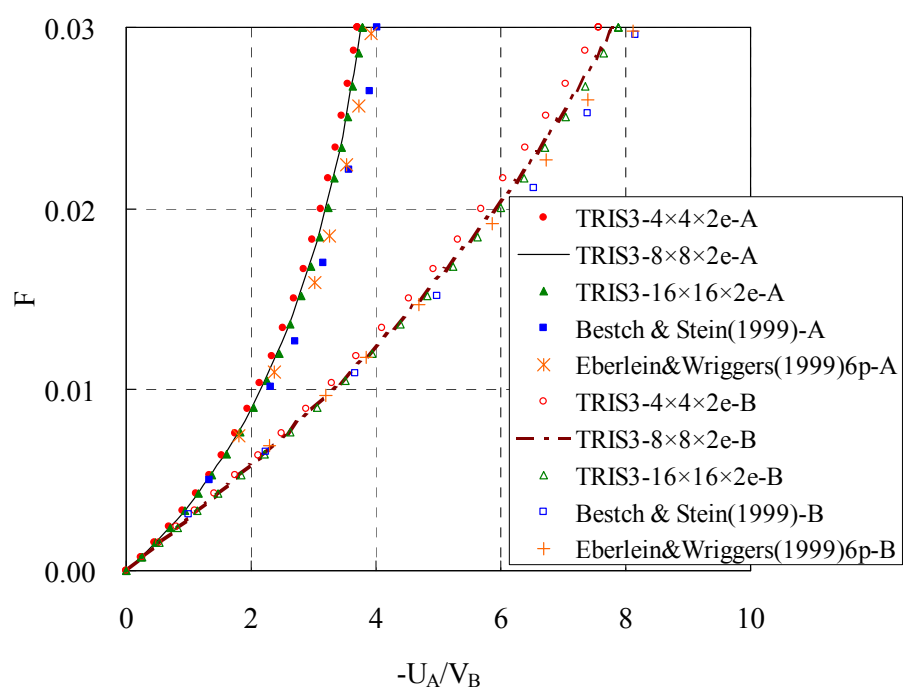

Figure 18. Load-deflection Curves at Pinching Points A and B of hemispherical shell

To illustrate the convergence characteristics of the TRIS3 element, the deflections at the pinched points $\mathrm{A}$ and $\mathrm{B}$ of the hemispherical shell under $\mathrm{F}=0.03$ are presented in Table 5 , with the relative errors based on the results from the highest-density $24 \times 24 \times 2$ mesh.

Table 5. Deflection at the Pinched Point A and B under $F=0.03$

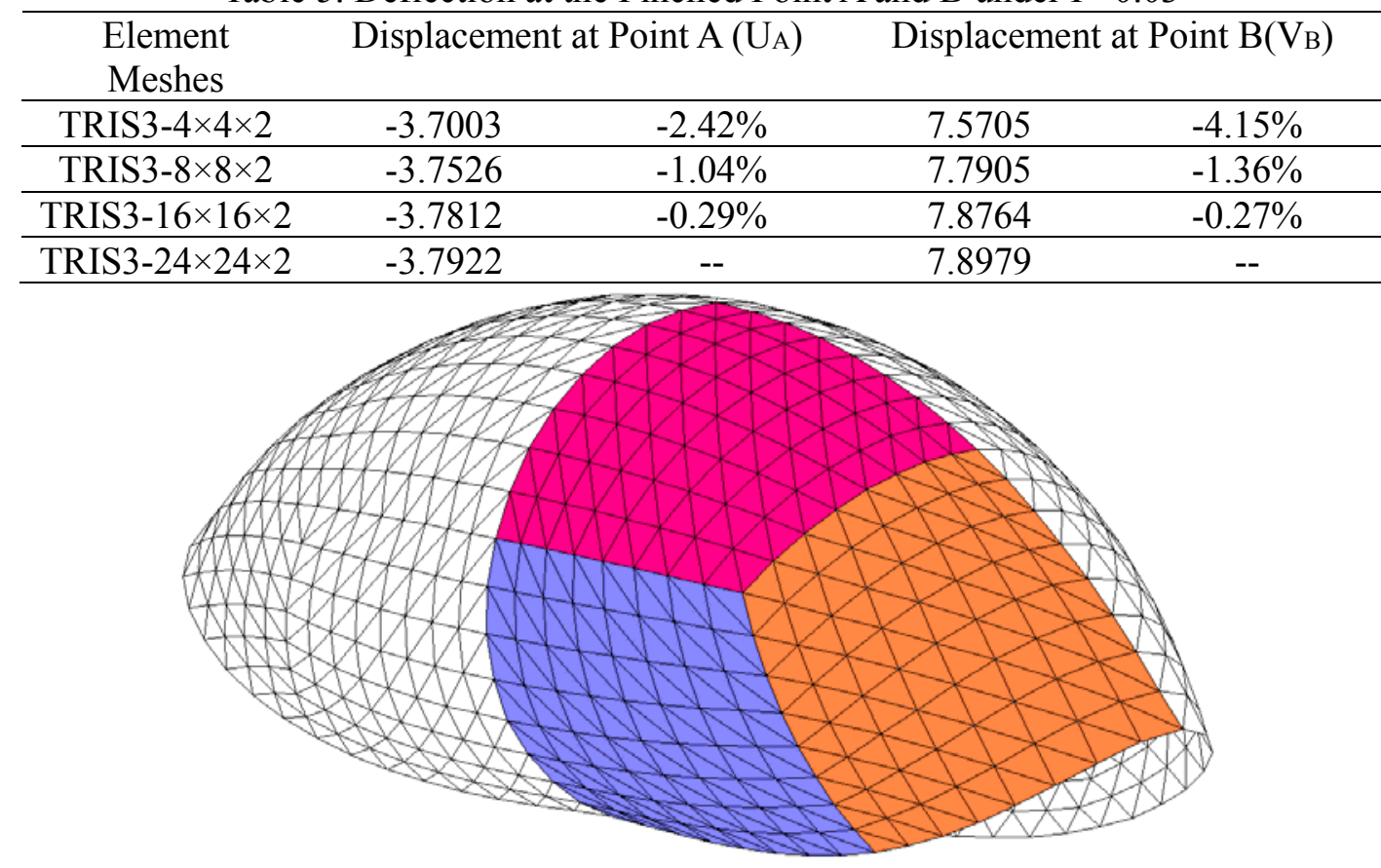

Figure 19. Deformed Shape of a Pinched Hemispherical Shell

The deformed shape of the complete hemispherical shell at the load level of $\mathrm{F}=0.03$ is presented in Figure 19. Plastic zone occurred in the pinched hemispherical shell at this load level. 


\subsection{Pinched Cylinder}

Consider a cylinder supported by two rigid diaphragms at its two ends, where only the displacement along the longitudinal axis is allowed (see Figure 20), with length $2 \mathrm{~L}=600$, radius $\mathrm{R}=300$, and thickness $\mathrm{h}=3$. The material properties are $\mathrm{E}=3000, \mu=0.3, \mathrm{f}_{\mathrm{y}}=24.3$ and $\mathrm{H}=300$. The cylinder is subjected to a pair of pinching concentrated loads (Figure 20).

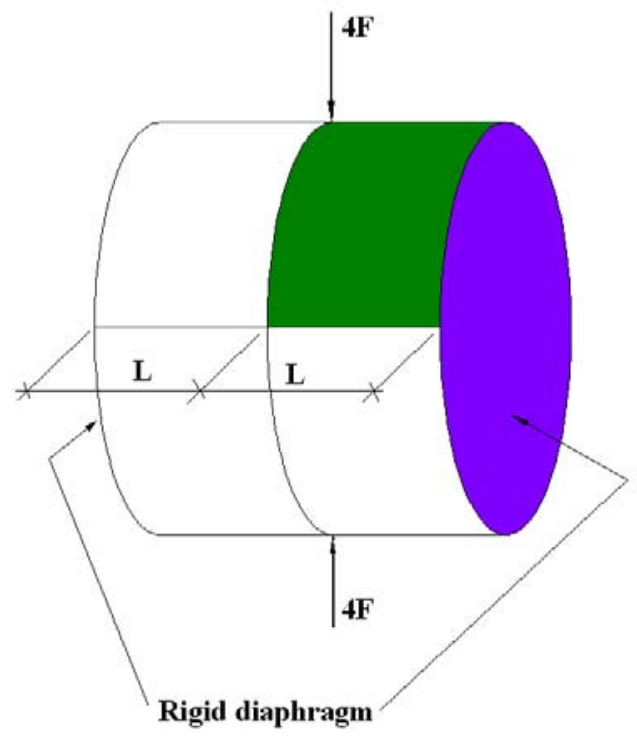

Figure 20. A Pinched Cylinder with Two End Diaphragms

Again, owing to symmetry, only one-eighth of the cylinder (the colored zone of Figure 20) is analyzed with three different uniform meshes: (a) $24 \times 24 \times 2$, (b) $32 \times 32 \times 2$ and (c) $48 \times 48 \times 2$ TRIS3 elements. The load-displacement curves at one pinched point are presented in Figure 21, where it can be seen that the results agree well with those from Eberlein \& Wriggers [78] using $32 \times 32$ four-node quadrilateral shell elements, Valente et al. [80] using $32 \times 32$ S4E6P5 shell elements, and Miehe [81] using 32×32 mixed brick-type shell elements.

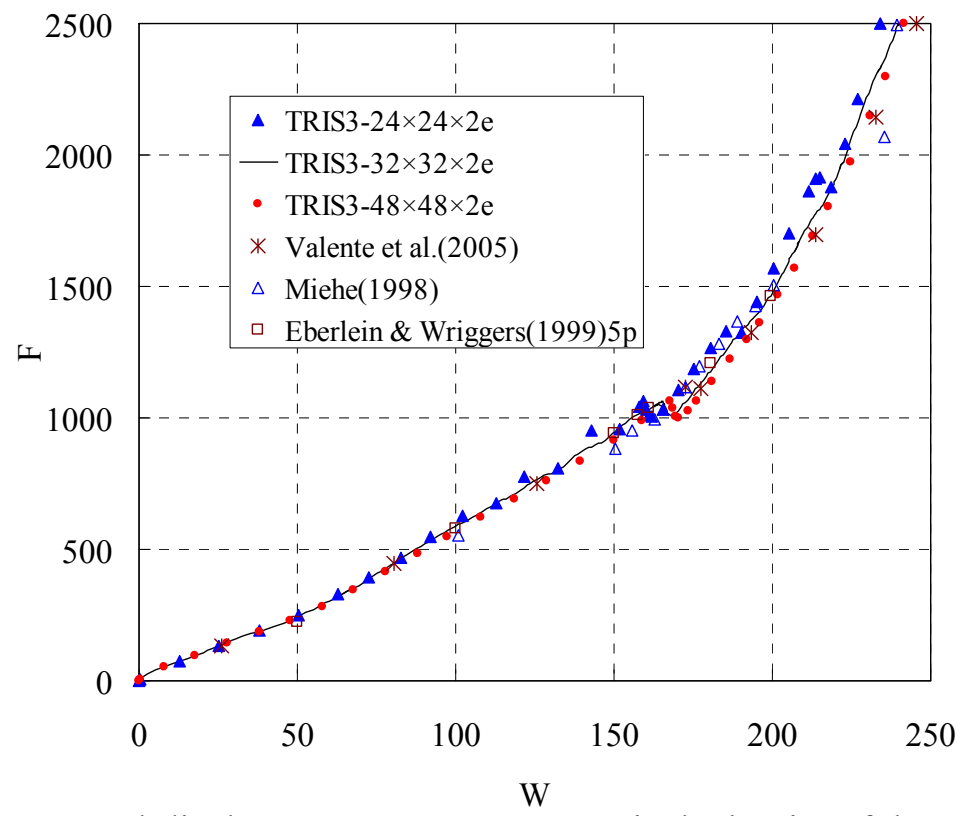

Figure 21. Load-displacement Curves at One Pinched Point of the Cylinder 
To evaluate the convergence of the TRIS3 element, the deflections at the pinched point of the cylinder under $\mathrm{F}=2500$ are presented in Table 6 , with the relative errors based on the results of the highest-density $48 \times 48 \times 2$ mesh.

Table 6. Deflection at the Pinched Point of the Cylinder

\begin{tabular}{ccc}
\hline Element meshes & Deflection at the loading point \\
\hline TRIS3-24 $\times 24 \times 2$ & 234.1663 & $-3.11 \%$ \\
\hline TRIS3-32 $\times 32 \times 2$ & 239.6611 & $-0.83 \%$ \\
\hline TRIS3-48 $\times 48 \times 2$ & 241.6718 & -- \\
\hline
\end{tabular}

The deformed shape of the overall cylinder obtained using $24 \times 24 \times 2$ TRIS3 elements at a load level $\mathrm{F}=2500$ is depicted in Figure 22, showing clear large displacements and large nodal rotations; plastic deformation occurs even at a very low loading level.

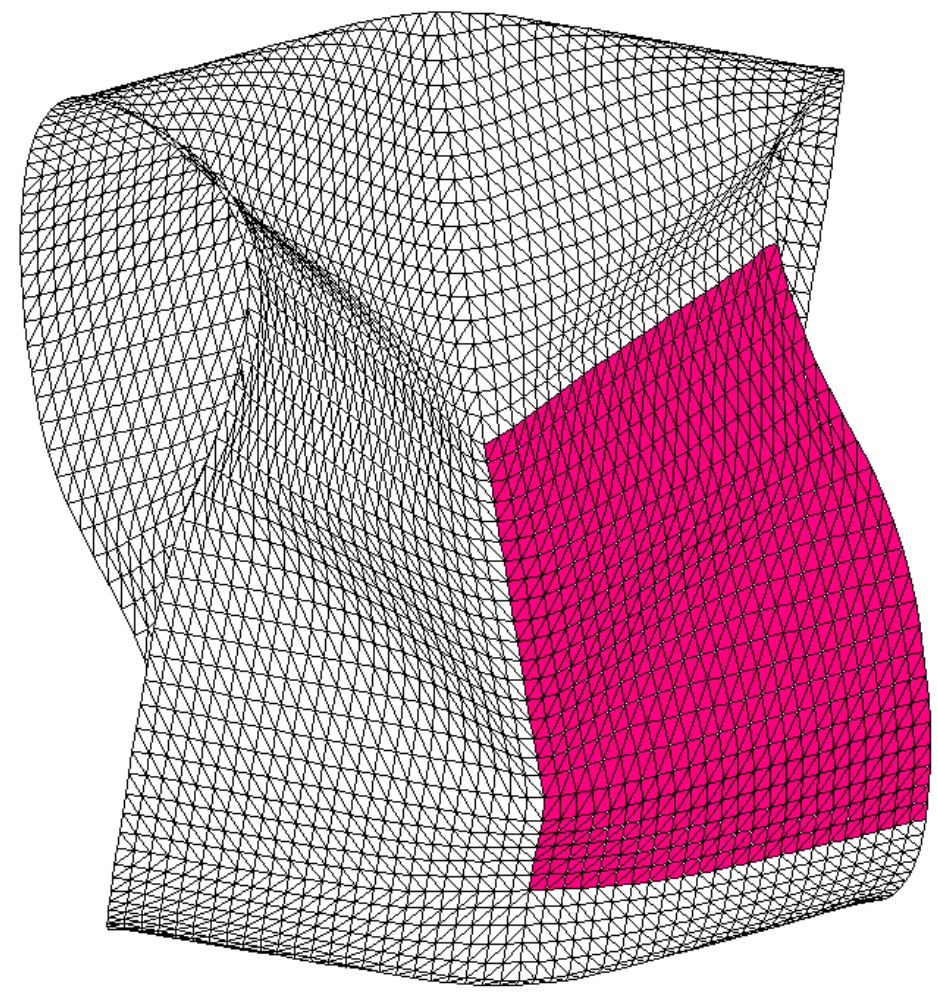

Figure 22. Deformed Shape of the Pinched Cylinder

\section{CONCLUSIONS}

A 3-node co-rotational triangular shell element formulation has been proposed for elasto-plastic plate/shell analysis involving large displacement and large rotation. To overcome the locking problems, assumed membrane strains and assumed shear strains calculated respectively from the edge-member membrane strains and the edge-member transverse shear strains by using the line integration methods are employed in the present element formulation. The residual bending flexibility is added to the transverse shear flexibility to improve further the accuracy of the element. The reliability and convergence of the present shell formulation are demonstrated in a number of problems that include linear elastic patch tests together with six elastic/elasto-plastic plate and shell problems involving large displacement and large rotation. 


\section{APPENDIX A: Various derivatives of strains with respect to local nodal variables}

The first derivatives of membrane strains with respect to local nodal variables:

$$
\begin{aligned}
& \mathbf{B}_{m}=\left\lfloor\begin{array}{llllll}
\mathbf{B}_{m 1} & \mathbf{0} & \mathbf{B}_{m 2} & \mathbf{0} & \mathbf{B}_{m 3} & \mathbf{0}
\end{array}\right\rfloor \\
& \mathbf{B}_{m i}=\left[\begin{array}{ccc}
N_{i, x} & 0 & \frac{\partial(w+z)}{\partial x} N_{i, x} \\
0 & N_{i, y} & \frac{\partial(w+z)}{\partial y} N_{i, y} \\
N_{i, y} & N_{i, x} & \frac{\partial(w+z)}{\partial y} N_{i, x}+\frac{\partial(w+z)}{\partial x} N_{i, y}
\end{array}\right] \quad i=1,2, \ldots, 3
\end{aligned}
$$

where,

$$
\begin{aligned}
& N_{i, x}=J_{11}^{-1} N_{i, \xi}+J_{12}^{-1} N_{i, \eta} \\
& N_{i, y}=J_{21}^{-1} N_{i, \xi}+J_{22}^{-1} N_{i, \eta}
\end{aligned}
$$

$J_{j k}^{-1}(j, k=1,2)$ is the component of inverse Jacobian matrix at $j t h$ row and $k t h$ column; $N_{i, \xi}$ and $N_{i, \eta}$ are respectively the first derivative of the shape function $N_{i}$ with respect to $\xi$ and $\eta$.

The first derivatives of assumed membrane strains with respect to local nodal variables:

$$
\begin{aligned}
& \widetilde{\mathbf{B}}_{m}=\mathbf{P} \boldsymbol{\Gamma}^{-1}\left[\mathbf{B}_{k}\right] \\
& \mathbf{B}_{k, 5 i-4: 5 i-3}=-\frac{\mathbf{a}_{i j 0}^{\mathrm{T}}(1: 2)}{\left|\mathbf{a}_{i j 0}\right|^{2}} \\
& \mathbf{B}_{k, 5 i-2}=-\frac{w_{j}-w_{i}}{\left|\mathbf{a}_{i j 0}\right|^{2}} \\
& \mathbf{B}_{k, 5 j-4: 5 j-3}=\frac{\mathbf{a}_{i j 0}^{\mathrm{T}}(1: 2)}{\left|\mathbf{a}_{i j 0}\right|^{2}} \\
& \mathbf{B}_{k, 5 j-2}=\frac{w_{j}-w_{i}}{\left|\mathbf{a}_{i j 0}\right|^{2}}
\end{aligned}
$$

where, the ordered triplets $(k, i, j)$ are given by $(\mathrm{A}, 1,2),(\mathrm{B}, 2,3)$ and $(\mathrm{C}, 3,1) ; \mathbf{a}_{i j 0}(1: 2)$ represents the sub-vector of the vector $\mathbf{a}_{i j 0}$ consisting of its first two components. 
The first derivatives of shear strains with respect to local nodal variables:

$$
\begin{aligned}
& \mathbf{B}_{s}=\left[\begin{array}{llllll}
\mathbf{B}_{s 1} & \mathbf{B}_{s 2} & \mathbf{B}_{s 3} & \mathbf{B}_{s 4} & \mathbf{B}_{s 5} & \mathbf{B}_{s 6}
\end{array}\right\rfloor \\
& \mathbf{B}_{s(2 i-1)}=\left[\begin{array}{lll}
0 & 0 & N_{i, x} \\
0 & 0 & N_{i, y}
\end{array}\right] \quad i=1,2,3 \\
& \mathbf{B}_{s(2 i)}=\left[\begin{array}{cc}
N_{i} & 0 \\
0 & N_{i}
\end{array}\right] \quad i=1,2,3
\end{aligned}
$$

The first derivatives of assumed shear strains with respect to local nodal variables:

$$
\begin{aligned}
& \widetilde{\mathbf{B}}_{s}=\mathbf{P} \boldsymbol{\Omega}^{-1}\left[\mathbf{B}_{s k} / \rho_{k}\right] \\
& \mathbf{B}_{s k, 5 i-2}=-\frac{1}{\left|\mathbf{a}_{i j 0}\right|} \\
& \mathbf{B}_{s k, 5 j-2}=\frac{1}{\left|\mathbf{a}_{i j 0}\right|} \\
& \mathbf{B}_{s k, 5 i-1: 5 i}=\frac{\mathbf{a}_{i j 0}^{\mathrm{T}}(1: 2)}{2\left|\mathbf{a}_{i j 0}\right|} \\
& \mathbf{B}_{s k, 5 j-1: 5 j}=\frac{\mathbf{a}_{i j 0}^{\mathrm{T}}(1: 2)}{2\left|\mathbf{a}_{i j 0}\right|}
\end{aligned}
$$

where, $\mathbf{B}_{s k}$ represents the first derivative of the edge-member transverse shear strain at Point $k$ $(k=\mathrm{A}, \mathrm{B}, \mathrm{C})$ with respect to local nodal variables; the ordered triplets $(k, i, j)$ in Eqs. (A-5a $\sim \mathrm{e})$ are given by $(\mathrm{A}, 1,2),(\mathrm{B}, 2,3)$ and $(\mathrm{C}, 3,1)$.

The first derivatives of bending strains with respect to local nodal variables:

$$
\begin{aligned}
& \mathbf{B}_{b}=\left\lfloor\begin{array}{lllllll}
\mathbf{0} & \mathbf{B}_{b 1} & \mathbf{0} & \mathbf{B}_{b 2} & \mathbf{0} & \mathbf{B}_{b 3}
\end{array}\right\rfloor \\
& \mathbf{B}_{b i}=\left[\begin{array}{cc}
N_{i, x} & 0 \\
0 & N_{i, y} \\
N_{i, y} & N_{i, x}
\end{array}\right] \quad i=1,2,3
\end{aligned}
$$

The second derivatives of membrane strains with respect to local nodal variables:

$$
\begin{aligned}
& \left(\frac{\partial \mathbf{B}_{m}}{\partial \mathbf{u}_{\mathrm{L}}^{\mathrm{T}}}\right)_{1,5 i-2,5 j-2}=N_{i, x} N_{j, x} \\
& \left(\frac{\partial \mathbf{B}_{m}}{\partial \mathbf{u}_{\mathrm{L}}^{\mathrm{T}}}\right)_{2,5 i-2,5 j-2}=N_{i, y} N_{j, y} \quad i, j=1,2,3 \\
& \left(\frac{\partial \mathbf{B}_{m}}{\partial \mathbf{u}_{\mathrm{L}}^{\mathrm{T}}}\right)_{3,5 i-2,5 j-2}=N_{i, x} N_{j, y}+N_{i, y} N_{j, x}
\end{aligned}
$$

where, three subscripts outside the parentheses in the left side of (A-7) represent the position of the component at the three dimensional matrix. The values of other unmentioned components of the three dimensional matrix are equal to zero.

The second derivatives of assumed membrane strains with respect to local nodal variables:

$$
\frac{\partial \widetilde{\mathbf{B}}_{m}}{\partial \mathbf{u}_{\mathrm{L}}^{\mathrm{T}}}=\mathbf{P} \boldsymbol{\Gamma}^{-1}\left[\frac{\partial \mathbf{B}_{k}}{\partial \mathbf{u}_{\mathrm{L}}^{\mathrm{T}}}\right], \quad k=\mathrm{A}, \mathrm{B}, \mathrm{C}
$$




$$
\begin{aligned}
& \left(\frac{\partial \mathbf{B}_{k}}{\partial \mathbf{u}_{\mathrm{L}}^{\mathrm{T}}}\right)_{5 i-2,5 i-2}=\left(\frac{\partial \mathbf{B}_{k}}{\partial \mathbf{u}_{\mathrm{L}}^{\mathrm{T}}}\right)_{5 j-2,5 j-2}=\frac{1}{\left|\mathbf{a}_{i j 0}\right|^{2}} \\
& \left(\frac{\partial \mathbf{B}_{k}}{\partial \mathbf{u}_{\mathrm{L}}^{\mathrm{T}}}\right)_{5 i-2,5 j-2}=\left(\frac{\partial \mathbf{B}_{k}}{\partial \mathbf{u}_{\mathrm{L}}^{\mathrm{T}}}\right)_{5 j-2,5 i-2}=-\frac{1}{\left|\mathbf{a}_{i j 0}\right|^{2}}
\end{aligned}
$$

APPENDIX B: Sub-matrices of transformation matrix $T$ and its first derivatives with respect to global nodal variables

Sub-matrices of transformation matrix $\mathbf{T}$ :

$$
\begin{aligned}
& \frac{\partial \mathbf{t}_{k}}{\partial \mathbf{d}_{l}^{\mathrm{T}}}=\frac{\partial \mathbf{R}}{\partial \mathbf{d}_{l}^{\mathrm{T}}}\left(\mathbf{d}_{k}+\mathbf{v}_{k 0}\right)+\mathbf{R} \delta_{k l} \mathbf{I}=\left(\frac{\partial}{\partial \mathbf{d}_{l}^{\mathrm{T}}}\left[\begin{array}{c}
\mathbf{e}_{x}^{\mathrm{T}} \\
\mathbf{e}_{y}^{\mathrm{T}} \\
\mathbf{e}_{z}^{\mathrm{T}}
\end{array}\right]\right)\left(\mathbf{d}_{k}+\mathbf{v}_{k 0}\right)+\mathbf{R} \delta_{k l} \mathbf{I} \\
& \frac{\partial \boldsymbol{\theta}_{k}}{\partial \mathbf{d}_{l}^{\mathrm{T}}}=\frac{\partial \mathbf{R}_{h}}{\partial \mathbf{d}_{l}^{\mathrm{T}}} \mathbf{p}_{k}=\left(\frac{\partial}{\partial \mathbf{d}_{l}^{\mathrm{T}}}\left[\begin{array}{c}
\mathbf{e}_{x}^{\mathrm{T}} \\
\mathbf{e}_{y}^{\mathrm{T}}
\end{array}\right]\right) \mathbf{p}_{k} \\
& \frac{\partial \boldsymbol{\theta}_{k}}{\partial \mathbf{n}_{g l}^{\mathrm{T}}}=\mathbf{R}_{h} \delta_{k l} \frac{\partial \mathbf{p}_{k}}{\partial \mathbf{n}_{g l}^{\mathrm{T}}}=\left[\begin{array}{c}
\mathbf{e}_{x}^{\mathrm{T}} \\
\mathbf{e}_{y}^{\mathrm{T}}
\end{array}\right] \delta_{k l} \frac{\partial \mathbf{p}_{k}}{\partial \mathbf{n}_{g l}^{\mathrm{T}}} \\
& \frac{\partial \mathbf{e}_{x}}{\partial \mathbf{d}_{l}^{\mathrm{T}}}=\left[\frac{\mathbf{I}}{\left|\mathbf{e}_{x}^{\prime}+\mathbf{e}_{y}^{\prime n}\right|}-\frac{\left(\mathbf{e}_{x}^{\prime}+\mathbf{e}_{y}^{\prime n}\right) \otimes\left(\mathbf{e}_{x}^{\prime}+\mathbf{e}_{y}^{\prime n}\right)}{\left|\mathbf{e}_{x}^{\prime}+\mathbf{e}_{y}^{\prime \prime}\right|^{3}}\right]\left(\frac{\partial \mathbf{e}_{x}^{\prime}}{\partial \mathbf{d}_{l}^{\mathrm{T}}}+\frac{\partial \mathbf{e}_{y}^{\prime n}}{\partial \mathbf{d}_{l}^{\mathrm{T}}}\right) \\
& \frac{\partial \mathbf{e}_{y}^{\prime n}}{\partial \mathbf{d}_{l}^{\mathrm{T}}}=\frac{\partial \mathbf{e}_{y}^{\prime}}{\partial \mathbf{d}_{l}^{\mathrm{T}}} \times \mathbf{e}_{z}+\mathbf{e}_{y}^{\prime} \times \frac{\partial \mathbf{e}_{z}}{\partial \mathbf{d}_{l}^{\mathrm{T}}} \\
& \frac{\partial \mathbf{e}_{x}^{\prime}}{\partial \mathbf{d}_{l}^{\mathrm{T}}}=a_{x 1} \frac{\partial \mathbf{v}_{12}}{\partial \mathbf{d}_{l}^{\mathrm{T}}} \\
& \frac{\partial \mathbf{e}_{y}^{\prime}}{\partial \mathbf{d}_{l}^{\mathrm{T}}}=a_{y 1} \frac{\partial \mathbf{v}_{12}}{\partial \mathbf{d}_{l}^{\mathrm{T}}}+a_{y 2} \frac{\partial \mathbf{v}_{23}}{\partial \mathbf{d}_{l}^{\mathrm{T}}}
\end{aligned}
$$

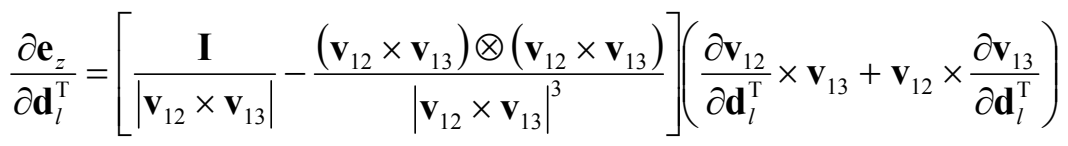

$$
\begin{aligned}
& \frac{\partial \mathbf{e}_{y}}{\partial \mathbf{d}_{l}^{\mathrm{T}}}=\frac{\partial \mathbf{e}_{z}}{\partial \mathbf{d}_{l}^{\mathrm{T}}} \times \mathbf{e}_{x}+\mathbf{e}_{z} \times \frac{\partial \mathbf{e}_{x}}{\partial \mathbf{d}_{l}^{\mathrm{T}}}
\end{aligned}
$$

In Eqs. B6-B8, $\frac{\partial \mathbf{v}_{12}}{\partial \mathbf{d}_{l}^{\mathrm{T}}}=-\mathbf{I}, l=1 ; \frac{\partial \mathbf{v}_{12}}{\partial \mathbf{d}_{l}^{\mathrm{T}}}=\mathbf{I}, l=2 ; \frac{\partial \mathbf{v}_{12}}{\partial \mathbf{d}_{l}^{\mathrm{T}}}=0, l=3 ; \frac{\partial \mathbf{v}_{13}}{\partial \mathbf{d}_{l}^{\mathrm{T}}}=-\mathbf{I}, l=1 ; \frac{\partial \mathbf{v}_{13}}{\partial \mathbf{d}_{l}^{\mathrm{T}}}=\mathbf{I}$,

$$
\begin{aligned}
& l=3 ; \frac{\partial \mathbf{v}_{13}}{\partial \mathbf{d}_{l}^{\mathrm{T}}}=0, \quad l=2 . \\
& \frac{\partial \mathbf{p}_{k}}{\partial \mathbf{n}_{g k}^{\mathrm{T}}}=\left[\begin{array}{ll}
\frac{\partial p_{k, X}}{\partial p_{k, n_{k}}} & \frac{\partial p_{k, X}}{\partial p_{k, m_{k}}} \\
\frac{\partial p_{k, Y}}{\partial p_{k, n_{k}}} & \frac{\partial p_{k, Y}}{\partial p_{k, m_{k}}} \\
\frac{\partial p_{k, Z}}{\partial p_{k, n_{k}}} & \frac{\partial p_{k, Z}}{\partial p_{k, m_{k}}}
\end{array}\right]
\end{aligned}
$$


where, $p_{k, X}, p_{k, Y}, p_{k, Z}$ are three components of the mid-surface normal vector $\mathbf{p}_{i}$ in the global coordinate axes $\mathrm{X}, \mathrm{Y}, \mathrm{Z}$ directions; $p_{k, n_{k}}, p_{k, m_{k}}$ are two vectorial rotational variables of Node $i$, they are the two smallest components among $p_{k, X}, p_{k, Y}, p_{k, Z} ; \frac{\partial p_{k, n_{k}}}{\partial p_{k, n_{k}}}=\frac{\partial p_{k, m_{k}}}{\partial p_{k, m_{k}}}=1$; $\frac{\partial p_{k, n_{k}}}{\partial p_{k, m_{k}}}=\frac{\partial p_{k, m_{k}}}{\partial p_{k, n_{k}}}=0 ; \frac{\partial p_{k, l_{k}}}{\partial p_{k, n_{k}}}=-\frac{p_{k, n_{k}}}{p_{k, l_{k}}}$ and $\frac{\partial p_{k, l_{k}}}{\partial p_{k, m_{k}}}=-\frac{p_{k, m_{k}}}{p_{k, l_{k}}}, l_{k} \neq n_{k} \neq m_{k}, l_{k}, n_{k}, m_{k} \in\{X, Y, Z\}$.

$\frac{\partial^{2} \mathbf{t}_{i}}{\partial \mathbf{d}_{j}^{\mathrm{T}} \partial \mathbf{u}_{G}^{\mathrm{T}}}=\left[\frac{\partial^{2} \mathbf{t}_{i}}{\partial \mathbf{d}_{j}^{\mathrm{T}} \partial \mathbf{d}_{1}^{\mathrm{T}}} \mathbf{0} \frac{\partial^{2} \mathbf{t}_{i}}{\partial \mathbf{d}_{j}^{\mathrm{T}} \partial \mathbf{d}_{2}^{\mathrm{T}}} \mathbf{0} \frac{\partial^{2} \mathbf{t}_{i}}{\partial \mathbf{d}_{j}^{\mathrm{T}} \partial \mathbf{d}_{3}^{\mathrm{T}}} \mathbf{0}\right]$

$\frac{\partial^{2} \boldsymbol{\theta}_{i}}{\partial \mathbf{d}_{j}^{\mathrm{T}} \partial \mathbf{u}_{G}^{\mathrm{T}}}=\left[\frac{\partial^{2} \boldsymbol{\theta}_{i}}{\partial \mathbf{d}_{j}^{\mathrm{T}} \partial \mathbf{d}_{1}^{\mathrm{T}}} \frac{\partial^{2} \boldsymbol{\theta}_{i}}{\partial \mathbf{d}_{j}^{\mathrm{T}} \partial \mathbf{n}_{g 1}^{\mathrm{T}}} \frac{\partial^{2} \boldsymbol{\theta}_{i}}{\partial \mathbf{d}_{j}^{\mathrm{T}} \partial \mathbf{d}_{2}^{\mathrm{T}}} \frac{\partial^{2} \boldsymbol{\theta}_{i}}{\partial \mathbf{d}_{j}^{\mathrm{T}} \partial \mathbf{n}_{g 2}^{\mathrm{T}}} \frac{\partial^{2} \boldsymbol{\theta}_{i}}{\partial \mathbf{d}_{j}^{\mathrm{T}} \partial \mathbf{d}_{3}^{\mathrm{T}}} \frac{\partial^{2} \boldsymbol{\theta}_{i}}{\partial \mathbf{d}_{j}^{\mathrm{T}} \partial \mathbf{n}_{g 3}^{\mathrm{T}}}\right]$

$\frac{\partial^{2} \boldsymbol{\theta}_{i}}{\partial \mathbf{n}_{g j}^{\mathrm{T}} \partial \mathbf{u}_{G}^{\mathrm{T}}}=\left[\frac{\partial^{2} \boldsymbol{\theta}_{i}}{\partial \mathbf{n}_{g j}^{\mathrm{T}} \partial \mathbf{d}_{1}^{\mathrm{T}}} \frac{\partial^{2} \boldsymbol{\theta}_{i}}{\partial \mathbf{n}_{g j}^{\mathrm{T}} \partial \mathbf{n}_{g 1}^{\mathrm{T}}} \frac{\partial^{2} \boldsymbol{\theta}_{i}}{\partial \mathbf{n}_{g j}^{\mathrm{T}} \partial \mathbf{d}_{2}^{\mathrm{T}}} \frac{\partial^{2} \boldsymbol{\theta}_{i}}{\partial \mathbf{n}_{g j}^{\mathrm{T}} \partial \mathbf{n}_{g 2}^{\mathrm{T}}} \frac{\partial^{2} \boldsymbol{\theta}_{i}}{\partial \mathbf{n}_{g j}^{\mathrm{T}} \partial \mathbf{d}_{3}^{\mathrm{T}}} \frac{\partial^{2} \boldsymbol{\theta}_{i}}{\partial \mathbf{n}_{g j}^{\mathrm{T}} \partial \mathbf{n}_{g 3}^{\mathrm{T}}}\right]$

$\frac{\partial^{2} \mathbf{t}_{i}}{\partial \mathbf{d}_{j}^{\mathrm{T}} \partial \mathbf{d}_{k}^{\mathrm{T}}}=\frac{\partial^{2} \mathbf{R}}{\partial \mathbf{d}_{j}^{\mathrm{T}} \partial \mathbf{d}_{k}^{\mathrm{T}}}\left(\mathbf{d}_{i}+\mathbf{v}_{i 0}\right)+\frac{\partial \mathbf{R}}{\partial \mathbf{d}_{j}^{\mathrm{T}}} \delta_{i k} \mathbf{I}+\frac{\partial \mathbf{R}}{\partial \mathbf{d}_{k}^{\mathrm{T}}} \delta_{i j} \mathbf{I}$

$=\left[\begin{array}{c}\frac{\partial^{2} \mathbf{e}_{x}^{\mathrm{T}}}{\partial \mathbf{d}_{j}^{\mathrm{T}} \partial \mathbf{d}_{k}^{\mathrm{T}}} \\ \frac{\partial^{2} \mathbf{e}_{y}^{\mathrm{T}}}{\partial \mathbf{d}_{j}^{\mathrm{T}} \partial \mathbf{d}_{k}^{\mathrm{T}}} \\ \frac{\partial^{2} \mathbf{e}_{z}^{\mathrm{T}}}{\partial \mathbf{d}_{j}^{\mathrm{T}} \partial \mathbf{d}_{k}^{\mathrm{T}}}\end{array}\right]\left(\mathbf{d}_{i}+\mathbf{v}_{i 0}\right)+\left[\begin{array}{c}\frac{\partial \mathbf{e}_{x}^{\mathrm{T}}}{\partial \mathbf{d}_{j}^{\mathrm{T}}} \\ \frac{\partial \mathbf{e}_{y}^{\mathrm{T}}}{\partial \mathbf{d}_{j}^{\mathrm{T}}} \\ \frac{\partial \mathbf{e}_{z}^{T}}{\partial \mathbf{d}_{j}^{\mathrm{T}}}\end{array}\right] \delta_{i k} \mathbf{I}+\left[\begin{array}{c}\frac{\partial \mathbf{e}_{x}^{\mathrm{T}}}{\partial \mathbf{d}_{k}^{\mathrm{T}}} \\ \frac{\partial \mathbf{e}_{y}^{\mathrm{T}}}{\partial \mathbf{d}_{k}^{\mathrm{T}}} \\ \frac{\partial \mathbf{e}_{z}^{\mathrm{T}}}{\partial \mathbf{d}_{k}^{\mathrm{T}}}\end{array}\right] \delta_{i j} \mathbf{I}$

$\frac{\partial^{2} \boldsymbol{\theta}_{i}}{\partial \mathbf{d}_{j}^{\mathrm{T}} \partial \mathbf{d}_{k}^{\mathrm{T}}}=\frac{\partial^{2} \mathbf{R}_{h}}{\partial \mathbf{d}_{j}^{\mathrm{T}} \partial \mathbf{d}_{k}^{\mathrm{T}}} \mathbf{p}_{i}=\left[\begin{array}{c}\frac{\partial^{2} \mathbf{e}_{x}^{\mathrm{T}}}{\partial \mathbf{d}_{j}^{\mathrm{T}} \partial \mathbf{d}_{k}^{\mathrm{T}}} \\ \frac{\partial^{2} \mathbf{e}_{y}^{\mathrm{T}}}{\partial \mathbf{d}_{j}^{\mathrm{T}} \partial \mathbf{d}_{k}^{\mathrm{T}}}\end{array}\right] \mathbf{p}_{i}$

$\frac{\partial^{2} \boldsymbol{\theta}_{i}}{\partial \mathbf{d}_{j}^{\mathrm{T}} \partial \mathbf{n}_{g k}^{\mathrm{T}}}=\frac{\partial \mathbf{R}_{h}}{\partial \mathbf{d}_{j}^{\mathrm{T}}} \delta_{i k} \frac{\partial \mathbf{p}_{i}}{\partial \mathbf{n}_{g k}^{\mathrm{T}}}=\left[\begin{array}{c}\frac{\partial \mathbf{e}_{x}^{\mathrm{T}}}{\partial \mathbf{d}_{j}^{\mathrm{T}}} \\ \frac{\partial \mathbf{e}_{y}^{\mathrm{T}}}{\partial \mathbf{d}_{j}^{\mathrm{T}}}\end{array}\right] \delta_{i k} \frac{\partial \mathbf{p}_{i}}{\partial \mathbf{n}_{g k}^{\mathrm{T}}}$

$\frac{\partial^{2} \boldsymbol{\theta}_{i}}{\partial \mathbf{n}_{g j}^{\mathrm{T}} \partial \mathbf{n}_{g k}^{\mathrm{T}}}=\mathbf{R}_{h} \delta_{i j} \delta_{i k} \frac{\partial^{2} \mathbf{p}_{i}}{\partial \mathbf{n}_{g j}^{\mathrm{T}} \partial \mathbf{n}_{g k}^{\mathrm{T}}}$

$\frac{\partial^{2} \mathbf{e}_{x}}{\partial \mathbf{d}_{j}^{\mathrm{T}} \partial \mathbf{d}_{k}^{\mathrm{T}}}=-\frac{\left(\mathbf{e}_{x}^{\prime}+\mathbf{e}_{y}^{\prime n}\right)^{\mathrm{T}}}{\left|\mathbf{e}_{x}^{\prime}+\mathbf{e}_{y}^{\prime n}\right|^{3}}\left(\frac{\partial \mathbf{e}_{x}^{\prime}}{\partial \mathbf{d}_{k}^{\mathrm{T}}}+\frac{\partial \mathbf{e}_{y}^{\prime n}}{\partial \mathbf{d}_{k}^{\mathrm{T}}}\right) \otimes\left(\frac{\partial \mathbf{e}_{x}^{\prime}}{\partial \mathbf{d}_{j}^{\mathrm{T}}}+\frac{\partial \mathbf{e}_{y}^{\prime n}}{\partial \mathbf{d}_{j}^{\mathrm{T}}}\right)+\frac{1}{\left|\mathbf{e}_{x}^{\prime}+\mathbf{e}_{y}^{\prime n}\right|} \frac{\partial^{2} \mathbf{e}_{y}^{\prime n}}{\partial \mathbf{d}_{j}^{\mathrm{T}} \partial \mathbf{d}_{k}^{\mathrm{T}}}$

$-\frac{1}{\left|\mathbf{e}_{x}^{\prime}+\mathbf{e}_{y}^{\left.\prime\right|^{3}}\right|^{3}}\left[\left(\frac{\partial \mathbf{e}_{x}^{\prime}}{\partial \mathbf{d}_{k}^{\mathrm{T}}}+\frac{\partial \mathbf{e}_{y}^{\prime n}}{\partial \mathbf{d}_{k}^{\mathrm{T}}}\right) \otimes\left(\mathbf{e}_{x}^{\prime}+\mathbf{e}_{y}^{\prime n}\right)+\left(\mathbf{e}_{x}^{\prime}+\mathbf{e}_{y}^{\prime n}\right) \otimes\left(\frac{\partial \mathbf{e}_{x}^{\prime}}{\partial \mathbf{d}_{k}^{\mathrm{T}}}+\frac{\partial \mathbf{e}_{y}^{\prime n}}{\partial \mathbf{d}_{k}^{\mathrm{T}}}\right)\right]\left(\frac{\partial \mathbf{e}_{x}^{\prime}}{\partial \mathbf{d}_{j}^{\mathrm{T}}}+\frac{\partial \mathbf{e}_{y}^{\prime n}}{\partial \mathbf{d}_{j}^{\mathrm{T}}}\right)$

$+\frac{3}{\left|\mathbf{e}_{x}^{\prime}+\mathbf{e}_{y}^{\prime n}\right|^{5}}\left[\left(\mathbf{e}_{x}^{\prime}+\mathbf{e}_{y}^{\prime n}\right)^{\mathrm{T}}\left(\frac{\partial \mathbf{e}_{x}^{\prime}}{\partial \mathbf{d}_{k}^{\mathrm{T}}}+\frac{\partial \mathbf{e}_{y}^{\prime n}}{\partial \mathbf{d}_{k}^{\mathrm{T}}}\right) \otimes\left(\mathbf{e}_{x}^{\prime}+\mathbf{e}_{y}^{\prime n}\right) \otimes\left(\mathbf{e}_{x}^{\prime}+\mathbf{e}_{y}^{\prime n}\right)\right]\left(\frac{\partial \mathbf{e}_{x}^{\prime}}{\partial \mathbf{d}_{j}^{\mathrm{T}}}+\frac{\partial \mathbf{e}_{y}^{\prime n}}{\partial \mathbf{d}_{j}^{\mathrm{T}}}\right)$ 


$$
\begin{aligned}
& \frac{\partial^{2} \mathbf{e}_{y}^{\prime n}}{\partial \mathbf{d}_{j}^{\mathrm{T}} \partial \mathbf{d}_{k}^{\mathrm{T}}}=\frac{\partial \mathbf{e}_{y}^{\prime}}{\partial \mathbf{d}_{j}^{\mathrm{T}}} \times \frac{\partial \mathbf{e}_{z}}{\partial \mathbf{d}_{k}^{\mathrm{T}}}+\frac{\partial \mathbf{e}_{y}^{\prime}}{\partial \mathbf{d}_{k}^{\mathrm{T}}} \times \frac{\partial \mathbf{e}_{z}}{\partial \mathbf{d}_{j}^{\mathrm{T}}}+\mathbf{e}_{y}^{\prime} \times \frac{\partial^{2} \mathbf{e}_{z}}{\partial \mathbf{d}_{j}^{\mathrm{T}} \partial \mathbf{d}_{k}^{\mathrm{T}}} \\
& \frac{\partial^{2} \mathbf{e}_{z}}{\partial \mathbf{d}_{j}^{\mathrm{T}} \partial \mathbf{d}_{k}^{\mathrm{T}}}=\left[\frac{\mathbf{I}}{\left|\mathbf{v}_{12} \times \mathbf{v}_{13}\right|}-\frac{\left(\mathbf{v}_{12} \times \mathbf{v}_{13}\right) \otimes\left(\mathbf{v}_{12} \times \mathbf{v}_{13}\right)}{\left|\mathbf{v}_{12} \times \mathbf{v}_{13}\right|^{3}}\right]\left(\frac{\partial \mathbf{v}_{12}}{\partial \mathbf{d}_{j}^{\mathrm{T}}} \times \frac{\partial \mathbf{v}_{13}}{\partial \mathbf{d}_{k}^{\mathrm{T}}}+\frac{\partial \mathbf{v}_{12}}{\partial \mathbf{d}_{k}^{\mathrm{T}}} \times \frac{\partial \mathbf{v}_{13}}{\partial \mathbf{d}_{j}^{\mathrm{T}}}\right) \\
& -\left(\frac{\partial \mathbf{v}_{12}}{\partial \mathbf{d}_{j}^{\mathrm{T}}} \times \mathbf{v}_{13}+\mathbf{v}_{12} \times \frac{\partial \mathbf{v}_{13}}{\partial \mathbf{d}_{j}^{\mathrm{T}}}\right) \otimes\left[\frac{\left(\mathbf{v}_{12} \times \mathbf{v}_{13}\right)^{\mathrm{T}}}{\left|\mathbf{v}_{12} \times \mathbf{v}_{13}\right|^{3}}\left(\frac{\partial \mathbf{v}_{12}}{\partial \mathbf{d}_{k}^{\mathrm{T}}} \times \mathbf{v}_{13}+\mathbf{v}_{12} \times \frac{\partial \mathbf{v}_{13}}{\partial \mathbf{d}_{k}^{\mathrm{T}}}\right)\right] \\
& -\frac{\left(\mathbf{v}_{12} \times \mathbf{v}_{13}\right)}{\left|\mathbf{v}_{12} \times \mathbf{v}_{13}\right|^{3}} \otimes\left[\left(\frac{\partial \mathbf{v}_{12}}{\partial \mathbf{d}_{j}^{\mathrm{T}}} \times \mathbf{v}_{13}+\mathbf{v}_{12} \times \frac{\partial \mathbf{v}_{13}}{\partial \mathbf{d}_{j}^{\mathrm{T}}}\right)^{\mathrm{T}}\left(\frac{\partial \mathbf{v}_{12}}{\partial \mathbf{d}_{k}^{\mathrm{T}}} \times \mathbf{v}_{13}+\mathbf{v}_{12} \times \frac{\partial \mathbf{v}_{13}}{\partial \mathbf{d}_{k}^{\mathrm{T}}}\right)\right] \\
& -\left(\frac{\partial \mathbf{v}_{12}}{\partial \mathbf{d}_{k}^{\mathrm{T}}} \times \mathbf{v}_{13}+\mathbf{v}_{12} \times \frac{\partial \mathbf{v}_{13}}{\partial \mathbf{d}_{k}^{\mathrm{T}}}\right) \otimes\left[\frac{\left(\mathbf{v}_{12} \times \mathbf{v}_{13}\right)^{\mathrm{T}}}{\left|\mathbf{v}_{12} \times \mathbf{v}_{13}\right|^{3}}\left(\frac{\partial \mathbf{v}_{12}}{\partial \mathbf{d}_{j}^{\mathrm{T}}} \times \mathbf{v}_{13}+\mathbf{v}_{12} \times \frac{\partial \mathbf{v}_{13}}{\partial \mathbf{d}_{j}^{\mathrm{T}}}\right)\right] \\
& +\frac{3\left(\mathbf{v}_{12} \times \mathbf{v}_{13}\right)}{\left|\mathbf{v}_{12} \times \mathbf{v}_{13}\right|^{5}} \otimes\left[\left(\mathbf{v}_{12} \times \mathbf{v}_{13}\right)^{\mathrm{T}}\left(\frac{\partial \mathbf{v}_{12}}{\partial \mathbf{d}_{j}} \times \mathbf{v}_{13}+\mathbf{v}_{12} \times \frac{\partial \mathbf{v}_{13}}{\partial \mathbf{d}_{j}}\right)\right] \otimes\left[\left(\mathbf{v}_{12} \times \mathbf{v}_{13}\right)^{\mathrm{T}}\left(\frac{\partial \mathbf{v}_{12}}{\partial \mathbf{d}_{k}} \times \mathbf{v}_{13}+\mathbf{v}_{12} \times \frac{\partial \mathbf{v}_{13}}{\partial \mathbf{d}_{k}}\right)\right] \\
& \text { (B20) } \\
& \frac{\partial^{2} \mathbf{e}_{y}}{\partial \mathbf{d}_{j}^{\mathrm{T}} \partial \mathbf{d}_{k}^{\mathrm{T}}}=\frac{\partial^{2} \mathbf{e}_{z}}{\partial \mathbf{d}_{j}^{\mathrm{T}} \partial \mathbf{d}_{k}^{\mathrm{T}}} \times \mathbf{e}_{x}+\mathbf{e}_{z} \times \frac{\partial^{2} \mathbf{e}_{x}}{\partial \mathbf{d}_{j}^{\mathrm{T}} \partial \mathbf{d}_{k}^{\mathrm{T}}}+\frac{\partial \mathbf{e}_{z}}{\partial \mathbf{d}_{j}^{\mathrm{T}}} \times \frac{\partial \mathbf{e}_{x}}{\partial \mathbf{d}_{k}^{\mathrm{T}}}+\frac{\partial \mathbf{e}_{z}}{\partial \mathbf{d}_{k}^{\mathrm{T}}} \times \frac{\partial \mathbf{e}_{x}}{\partial \mathbf{d}_{j}^{\mathrm{T}}} \\
& \frac{\partial^{2} \mathbf{p}_{i}}{\partial \mathbf{n}_{g i}^{\mathrm{T}^{2}}}=\left[\begin{array}{cc}
\frac{\partial^{2} \mathbf{p}_{i}}{\partial p_{i, n_{i}}^{2}} & \frac{\partial^{2} \mathbf{p}_{i}}{\partial p_{i, n_{i}} \partial p_{i, m_{i}}} \\
\frac{\partial^{2} \mathbf{p}_{i}}{\partial p_{i, m_{i}} \partial p_{i, n_{i}}} & \frac{\partial^{2} \mathbf{p}_{i}}{\partial p_{i, m_{i}}^{2}}
\end{array}\right] \\
& \frac{\partial^{2} \mathbf{p}_{i}}{\partial p_{i, n_{i}}^{2}}=\left\{\begin{array}{l}
\frac{\partial^{2} p_{i, X}}{\partial p_{i, n_{i}}^{2}} \\
\frac{\partial^{2} p_{i, Y}}{\partial p_{i, n_{i}}^{2}} \\
\frac{\partial^{2} p_{i, Z}}{\partial p_{i, n_{i}}^{2}}
\end{array}\right\}, \quad \frac{\partial^{2} \mathbf{p}_{i}}{\partial p_{i, m_{i}}^{2}}=\left\{\begin{array}{l}
\frac{\partial^{2} p_{i, X}}{\partial p_{i, m_{i}}^{2}} \\
\frac{\partial^{2} p_{i, Y}}{\partial p_{i, m_{i}}^{2}} \\
\frac{\partial^{2} p_{i, Z}}{\partial p_{i, m_{i}}^{2}}
\end{array}\right\}, \quad \frac{\partial^{2} \mathbf{p}_{i}}{\partial p_{i, n_{i}} \partial p_{i, m_{i}}}=\left\{\begin{array}{l}
\frac{\partial^{2} p_{i, X}}{\partial p_{i, n_{i}} \partial p_{i, m_{i}}} \\
\frac{\partial^{2} p_{i, Y}}{\partial p_{i, n_{i}} \partial p_{i, m_{i}}} \\
\frac{\partial^{2} p_{i, Z}}{\partial p_{i, n_{i}} \partial p_{i, m_{i}}}
\end{array}\right\} \\
& \frac{\partial^{2} p_{i, l_{i}}}{\partial p_{i, n_{i}}^{2}}=-\frac{1}{p_{i, l_{i}}}-\frac{p_{i, n_{i}}^{2}}{p_{i, l_{i}}^{3}}, \frac{\partial^{2} p_{i, l_{i}}}{\partial p_{i, m_{i}}^{2}}=-\frac{1}{p_{i, l_{i}}}-\frac{p_{i, m_{i}}^{2}}{p_{i, l_{i}}^{3}}, \frac{\partial^{2} p_{i, l_{i}}}{\partial p_{i, n_{i}} \partial p_{i, m_{i}}}=-\frac{p_{i, n_{i}} p_{i, m_{i}}}{p_{i, l_{i}}^{3}}
\end{aligned}
$$

where, $p_{i, l_{i}}$ is the biggest component of Vector $\mathbf{p}_{i}$ in the global coordinate axes $\mathrm{X}, \mathrm{Y}, \mathrm{Z}$ directions; the second derivatives of other two components with respect to the vectorial rotational variables are equal to zero.

$p_{i, l_{i}}=s_{1} \sqrt{1-p_{i, n_{i}}^{2}-p_{i, m_{i}}^{2}} \quad i=1,2,3$

$s_{1}$ is equal to 1 or -1 . In an incremental solution procedure, it has the same sign as $p_{i, l_{i}}$ of the last incremental step, and its value will be updated after each incremental loading step. 


\section{ACKNOWLEDGMENTS}

This work is financed by the support program of the National Twelfth Five-Year-Plan for Science \& Technology (2012BAJ13B04).

\section{REFERENCES}

[1] Jeon, H.M., Lee, Y.Y., Lee, P.S. and Bathe, K.J., "The MITC3+ Shell Element in Geometric Nonlinear Analysis", Computers and Structures, 2015, Vol. 146, pp. 91-104.

[2] Lee, Y.Y., Lee, P.S., and Bathe, K.J., "The MITC3+ Shell Element and its Performance, Computers and Structures, 2014, Vol. 138, pp. 12-23.

[3] Jeon, H.M., Lee, P.S. and Bathe, K.J., "The MITC3 Shell Finite Element Enriched by Interpolation Covers", Computers and Structures, 2014, Vol. 134, pp. 128-142.

[4] Lee, P. S. and Bathe, K. J., "Development of MITC Isotropic Triangular Shell Finite Elements", Computers and Structures, 2004, Vol. 82, No. 11-12, pp. 945-962.

[5] Lee, P.S. and Bathe, K. J., "The Quadratic MITC Plate and MITC Shell Elements in Plate Bending", Advances in Engineering Software, 2010, Vol. 41, No. 5, pp. 712-728.

[6] Laulusa, A., Bauchau, O.A., Choi, J.Y., Tan, V.B.C., and Li, L., "Evaluation of Some Shear Deformable Shell Elements", International Journal of Solids and Structures, 2006, Vol. 43, No. 17, pp. 5033-5054.

[7] Chapelle, D. and Suarez, I.P., "Detailed Reliability Assessment of Triangular MITC Elements for Thin Shells", Computers and Structures, 2008, Vol. 86, No. 23-24, pp. 2192-2202.

[8] Lee, P.S., Noh, H.C. and Bathe, K.J., "Insight into 3-node Triangular Shell Finite Elements: The Effects of Element Isotropy and Mesh Patterns", Computers and Structures, 2007, Vol. 85, No. 7-8, pp. 404-418.

[9] Mohan, P. and Kapania, R.K., "Updated Lagrangian Formulation of a Flat Triangular Element for Thin Laminated Shells", AIAA Journal, 1998, Vol. 36, No. 2, pp. 273-281.

[10] Providasa, E. and Kattis, M.A., "An Assessment of Two Fundamental Flat Triangular Shell Elements with drilling Rotations", Computers and Structures, 2000, Vol. 77, No. 2, pp. 129-139.

[11] Khosravi, P., Ganesan, R. and Sedaghati, R., "Corotational Non-linear Analysis of Thin Plates and Shells using a New Shell Element", International Journal for Numerical Methods in Engineering, 2007, Vol. 69, No. 4, pp. 859-885.

[12] Khosravi, P., Ganesan, R. and Sedaghati, R., "An Efficient Facet Shell Element for Corotational Nonlinear Analysis of Thin and Moderately Thick Laminated Composite Structures", Computers and Structures, 2008, Vol. 86, No. 9, pp. 50-858.

[13] Carpenter, N., Stolarski, H. and Belytschko, T., "Improvements in 3-Node Triangular Shell Elements", International Journal for Numerical Methods in Engineering, 1986, Vol. 23, No. 9, pp. 1643-1667.

[14] Sabourin, F. and Brunet, M., "Analysis of Plates and Shells with a Simplified 3-node Triangular Element”, Thin-Walled Structures, 1995, Vol. 21, No. 3, pp. 209-223.

[15] Sabourin, F., and Brunet, M., "Detailed Formulation of the Rotation-free Triangular Element "S3" for General Purpose Shell Analysis", Engineering Computations, 2006, Vol. 23, No. 5-6, pp. 469-502.

[16] Oñate, E. and Zárate, F., "Rotation-free Triangular Plate and Shell Elements", International Journal for Numerical Methods in Engineering, 2000, Vol. 47, No. 1-3, pp. 557-603.

[17] Flores, F.G. and Oñate, E., "A Basic Thin Shell Triangle with Only Translational DOFs Large Strain Plasticity”, International Journal for Numerical Methods in Engineering, 2001, Vol. 51, No. 1, pp. 57-83. 
[18] Oñate, E. and Flores, F.G., "Advances in the Formulation of the Rotation-free Basic Shell Triangle", Computer Methods in Applied Mechanics and Engineering, 2005, Vol. 194, No. 21-24, pp. 2406-2443.

[19] Flores, F.G. and Oñate, E., "Improvements in the Membrane Behaviour of the Three Node Rotation-free BST Shell Triangle using an Assumed Strain Approach", Computer Methods in Applied Mechanics and Engineering, 2005, Vol. 194, No. 6-8, pp. 907-932.

[20] Oñate, E. and Zárate, F., "Extended Rotation-free Plate and Beam Elements with Shear Deformation Effects", International Journal for Numerical Methods in Engineering, 2010, Vol. 83, No. 2, pp. 196-227.

[21] Zárate, F. and Oñate., E., "Extended Rotation-free Shell Triangles with Transverse Shear Deformation Effects", Computational Mechanics, 2012, Vol. 49, No. 4, pp. 487-503.

[22] Stolarski, H., Gilmanov, A. and Sotiropoulos, F., "Nonlinear Rotation-free Three-node Shell Finite Element Formulation", International Journal for Numerical Methods in Engineering2013, Vol. 95, No. 9, pp. 740-770.

[23] Kabir, H. R. H., "A Shear-locking Free Robust Isoparametric 3-node Triangular Element for General Shells", Computers and Structures, 1994, Vol. 51, No. 4, pp. 425-436.

[24] Wu, S., Li, G.Y. and Belytschko, T., "A DKT Shell Element for Dynamic Large Deformation Analysis", Communications in Numerical Methods in Engineering, 2005, Vol. 21, No. 11, pp. 651-674.

[25] Kuznetsov, V.V. and Levyakov, S.V., "Geometrically Nonlinear Shell Finite Element based on the Geometry of a Planar Curve", Finite Elements in Analysis and Design, 2008, Vol. 44, No. 8, pp. 450-461.

[26] Kolahi, A.S. and Crisfield, M.A., "A Large-strain Elasto-plastic Shell Formulation using the Morley Triangle", International Journal for Numerical Methods in Engineering, 2001, Vol. 52, No. 8, pp. 829-849.

[27] Providas, E. and Kattis, M.A., A" Simple Finite Element Model for the Geometrically Nonlinear Analysis of Thin Shells", Computational Mechanics, 1999, Vol. 24, No. 2, pp. 127-137.

[28] Carpenter, N. and Stolarski, H. and Belytschko, T., "A Flat Triangular Shell Element with Improved Membrane Interpolation”, Communications in Applied Numerical Methods, 1985, Vol. 1, No. 4, pp. 161-168.

[29] Fish, J. and Belytschko, T., "Stabilized Rapidly Convergent 18-degrees-of-freedom Flat Shell Triangular Element", International Journal for Numerical Methods in Engineering, 1992, Vol. 33, No. 1, pp. 149-162.

[30] Allman, D.J., "On the assumed displacement-fields of a shallow curved shell finite-element", Communications in Numerical Methods in Engineering, 1995, Vol. 11, No. 2, pp. 159-166.

[31] To, C. W. S. and Wang, B., "Hybrid Strain-based Three-node Flat Triangular Laminated Composite Shell Elements", Finite Elements in Analysis and Design, 1998, Vol. 28, No. 3, pp. 177-207.

[32] Fajman, P., "New Triangular Plane Element with Drilling Degrees of Freedom", Journal of Engineering Mechanics-ASCE, 2002, Vol. 128, No. 4, pp. 413-418.

[33] Cai, Y.C. and Atluri, S.N., "Large Rotation Analyses of Plate/Shell Structures based on the Primal Variational Principle and a Fully Nonlinear Theory in the Updated Lagrangian Co-rotational Reference Frame", CMES-Computer Modeling in Engineering \& Sciences, 2012, Vol. 83, No. 3, pp. 249-273.

[34] Zhang, Y.X. and Cheung, Y.K., "A Refined Non-linear Non-conforming Triangular Plate/Shell Element", International Journal for Numerical Methods in Engineering, 2003, Vol. 56, No. 15, pp. 2387-2408.

[35] Poulsen, P. and Damkilde, L., "A Flat Triangular Shell Element with Loof Nodes", International Journal for Numerical Methods in Engineering, 1996, Vol. 39, No. 22, pp. 
3867-3887.

[36] Campello, E. M. B., Pimenta, P. M. and Wriggers, P., "A Triangular Finite Shell Element based on a Fully Nonlinear Shell Formulation", Computational Mechanics, 2003, Vol. 31, No. 6, pp. 505-518.

[37] Yang, H. T. Y., Saigal, S., Masud, A. and Kapania, R. K., "Survey of Recent Shell Finite Elements, International Journal for Numerical Methods in Engineering, 2000, Vol. 47, No. 1-3, pp. 101-127.

[38] Reissner, E., “A Note on Variational Theorems in Elasticity”, International Journal of Solids and Structures, 1965, Vol. 1, No. 1, pp. 93-95.

[39] Hughes, T. J. R. and Brezzi, F., "On Drilling Degrees of Freedom”, Computer Methods in Applied Mechanics and Engineering, 1989, Vol. 72, No. 1, pp. 105-121.

[40] Hughes, T. J. R., Masud, A. and Harari, I., "Numerical Assessment of Some Membrane Elements with Drilling Degrees of Freedom", Computers and Structures, 1995, Vol. 55, No. 2, pp. 297-314.

[41] Hughes, T. J. R., Masud, A. and Harari, I., "Dynamic Analysis with Drilling Degrees of Freedom", International Journal for Numerical Methods in Engineering, 1995, Vol. 38, No. 19, pp. 3193-3210.

[42] MacNeal, R.H., "Derivation of Element Stiffness Matrices by Assumed Strain Distribution", Nuclear Engineering and Design, 1982, Vol. 70, No. 1, pp. 3-12.

[43] Liu, M. L. and To, C. W. S., "Hybrid Strain-based 3-node Flat Triangular Shell Elements”, 1. Nonlinear-theory and Incremental Formulation", Computers and Structures, 1995, Vol. 54, No. 6, pp. 1031-1056.

[44] Liu, M. L. and To, C. W. S., "A Further Study of Hybrid Strain-based Three-node Flat Triangular Shell Elements", Finite Elements in Analysis and Design, 1998, Vol. 31, No. 2, pp. 135-152.

[45] To, C. W. S. and Wang, B., "Hybrid Strain-based Three-node Flat Triangular Laminated Composite Shell Elements", Finite Elements in Analysis and Design, 1998, Vol. 28, No. 3, pp. 177-207.

[46] Ibrahimbegovic, A. and Wilson, E.L., "A Modified Method of Incompatible Modes", Communications in Applied Numerical Methods, 1991, Vol. 7, No. 3, pp. 187-194.

[47] Bischoff, M. and Romero, I., "A Generalization of the Method of Incompatible Modes", International Journal for Numerical Methods in Engineering, 2007, Vol. 69, No. 9, pp. 1851-1868.

[48] Liu, W.K., Belytschko, T., Ong, J. S. J. and Law, S. E., "Use of Stabilization Matrices in Non-linear Analysis”, Engineering Computations, 1985, Vol. 2, No. 1, pp. 47-55.

[49] Belytschko, T., Wong, B.L. and Stolarski, H., "Assumed Strain Stabilization Procedure for the 9-node Lagrange Shell Element", International Journal for Numerical Methods in Engineering, 1989, Vol. 28, No. 2, pp. 385-414.

[50] Liu, W. K., Ong, J. S. J. and Uras, R. A., "Finite-element Stabilization Matrices - A Unification Approach", Computer Methods in Applied Mechanics and Engineering, 1985, Vol. 53, No. 1, pp. 13-46.

[51] Belytschko, T., Tsay, C.S. and Liu, W.K., "A Stabilization Matrix for the Bilinear Mindlin Plate Element”, Computer Methods in Applied Mechanics and Engineering, 1981, Vol. 29, No. 3, pp. 313-327.

[52] Masud, A., Tham, C.L., and Liu, W.K., "A Stabilized 3-D Co-rotational Formulation for Geometrically Nonlinear Analysis of Multi-layered Composite Shells", Computational Mechanics 2000, Vol. 26, No. 1, pp. 1-12.

[53] Belytschko, T. and Leviathan, I., "Physical Stabilization of the 4-node Shell Element with One-point Quadrature", Computer Methods in Applied Mechanics and Engineering, 1994, Vol. 113, No. 3-4, pp. 321-350. 
[54] Nguyen-Xuan, H., Liu, G. R. and Thai-Hoang, C. and Nguyen-Thoi, T., “An Edge-based Smoothed Finite Element Method (ES-FEM) with Stabilized Discrete Shear Gap Technique for Analysis of Reissner-Mindlin Plates", Computer Methods in Applied Mechanics and Engineering, 2010, Vol. 199, No. 9-12, pp. 471-489.

[55] Argyris, J. H., Papadrakakis, M., Apostolopoulou, C. and Koutsourelakis, S., "The TRIC Shell Element: Theoretical and Numerical Investigation", Computer Methods in Applied Mechanics and Engineering 2000, Vol. 182, No. 1-2, pp. 217-245.

[56] Ibrahimbegovic, A., "Finite Elastoplastic Deformations of Space-Curved Membranes", Computer Methods in Applied Mechanics and Engineering, 1994, Vol. 119, No. 3-4, pp. 371-394.

[57] El-Metwally, S. E., El-Shahhat, A. M. and Chen, W. F., "3-D Nonlinear-analysis of R/C Slender Columns”, Computers and Structures, 1990, Vol. 37, No. 5, pp. 863-872.

[58] Izzuddin, B. A. and Elnashai, A.S., "Adaptive Space Frame Analysis .2. AD Distributed Plasticity Approach", Proceedings of the Institution of Civil Engineers-Structures and Buildings, 1993, Vol. 99, No. 3, pp. 317-326.

[59] Spacone, E., Filippou, F.C. and Taucer, F.F., "Fibre Beam-column Model for Non-linear Analysis of R/C Frames", 1. Formulation, Earthquake Engineering \& Structural Dynamics, 1996, Vol. 25, No. 7, pp. 711-725.

[60] Izzuddin, B.A. and Lloyd Smith, D., "Large-displacement Analysis of Elastoplastic Thin-walled Frames" 1. Formulation and Implementation, Journal of Structural Engineering-ASCE, 1996, Vol. 122, No. 8, pp. 905-914.

[61] Crisfield, M. A., "Nonlinear Finite Element Analysis of Solid and Structures", Vol 1: Essentials. Wiley: Chichester, 1991.

[62] Becker, M. and Hackenberg, H. P., "A Constitutive Model for Rate Dependent and Rate Independent inelasticity, Application to IN718, International Journal of Plasticity, 2011, Vol. 27, No. 4, pp. 596-619.

[63] Simo, J. C. and Hughes, T. J. R., "Computational Inelasticity. Springer: New York, 1998.

[64] Khan, A. S. and Huang, S. J., "Continuum Theory of Plasticity. John Wiley \& Sons, Inc: New York. 1995; P94.

[65] Izzuddin, B.A. and Liang, Y., "Bisector and Zero-macrospin Co-rotational Systems for Shell Elements", International Journal for Numerical Methods in Engineering, 2015, Vol. 105, No. 4, pp. 286-320.

[66] Nour-Omid, B. and Rankin, C.C., "Finite Rotation Analysis and Consistent Linearization using Projectors", Computer Methods in Applied Mechanics and Engineering, 1991, Vol. 93, No. 3, pp. 353-384.

[67] Simo, J. C. and Tarnow, N. "A New Energy and Momentum Conserving Algorithm for the Non-linear Dynamics of Shells", International Journal for Numerical Methods in Engineering, 1994, Vol. 37, No. 15, pp. 2527-2549.

[68] Simo, J.C., Rifai, M.S. and Fox, D.D., "On a Stress Resultant Geometrically Exact Shell-model .6. Conserving Algorithms for Nonlinear Dynamics”, International Journal for Numerical Methods in Engineering, 1992, Vol. 34, No. 1, pp. 117-164.

[69] Tan, X. G. and Vu-Quoc, L., "Efficient and Accurate Multilayer Solid-shell Element: Nonlinear Materials at Finite Strain", International Journal for Numerical Methods in Engineering 2005, Vol. 63, No. 15, pp. 2124-2170.

[70] Vu-Quoc, L. and Tan, X. G., "Optimal Solid Shells for Nonlinear Analyses of Multilayer Composites”, Part II: Dynamics, Computer Methods in Applied Mechanics and Engineering, 2003, Vol. 192, No. 9-10, pp. 1017-1059.

[71] Li, Z. X., Zhuo, X., Vu-Quoc, L., Izzuddin, B.A. and Wei, H.Y., "A 4-node Co-rotational Quadrilateral Elasto-plastic Shell Element using Vectorial Rotational Variables", International Journal for Numerical Methods in Engineering, 2013, Vol. 95, No. 3, pp. 181-211. 
[72] Li, Z. X., Xiang, Y., Izzuddin, B. A., Vu-Quoc, L., Zhuo, X. and Zhang, C.J., "A 6-node Co-rotational Triangular Elasto-plastic Shell Element", Computational Mechanics, 2015, Vol. 55, No. 5, pp. 837-859.

[73] MacNeal, R. H. and Harder, R. L., "Proposed Standard Set of Problems to Test Finite Element Accuracy”, Finite Elements in Analysis and Design, 1985, Vol. 1, No. 1, pp. 3-20.

[74] Buechter, N. and Ramm, E., "Shell Theory Versus Degeneration - A Comparison in Large Rotation Finite-element Analysis", International Journal for Numerical Methods in Engineering, 1992, Vol. 34, No. 1, pp. 39-59.

[75] Simo, J. C., Fox, D.D. and Rifai, M. S., "On a Stress Resultant Geometrically Exact Shell Model", Part III: Computational Aspects of the Nonlinear Theory", Computer Methods in Applied Mechanics and Engineering, 1990, Vol. 79, No. 1, pp. 21-70.

[76] Jiang, L. and Chernuka, M.W., "A Simple 4-noded Corotational Shell Element for Arbitrarily Large Rotations", Computers and Structures, 1994, Vol. 53, No. 5, pp. $1123-1132$.

[77] Sze, K.Y., Chan, W.K. and Pian, T. H. H., "An 8-node Hybrid-stress Solid-shell Element for Geometric Nonlinear Analysis of Elastic Shells", International Journal for Numerical Methods in Engineering, 2000, Vol. 55, No. 7, pp. 853-878.

[78] Eberlein, R. and Wriggers, P., "Finite Element Concepts for Finite Elastoplastic Strains and Isotropic Stress Response in Shells: Theoretical and Computational Analysis", Computer Methods in Applied Mechanics and Engineering, 1999, Vol. 171, No. 3-4, pp. 243-279.

[79] Betsch, P. and Stein, E., "Numerical Implementation of Multiplicative Elasto-plasticity into Assumed Strain Elements with Application to Shells at Large Strains", Computer Methods in Applied Mechanics and Engineering, 1999, Vol. 179, No. 3-4, pp. 215-245.

[80] Valente, R. A. F., Parente, M. P. L., Jorge, R. M. N., de Sa, J. M. A. C. and Gracio, J. J., "Enhanced Transverse Shear Strain Shell Formulation Applied to Large Elasto-plastic Deformation Problems", International Journal for Numerical Methods in Engineering, 2005, Vol. 62, No. 10, pp.1360-1398.

[81] Miehe, C., "A Theoretical and Computational Model for Isotropic Elastoplastic Stress Analysis in Shells at Large Strains", Computer Methods in Applied Mechanics and Engineering, 1998, Vol. 155, No. 3-4, pp. 193-233.

[82] Izzuddin, B. A., "An Enhanced Co-rotational Approach for Large Displacement Analysis of Plates”, International Journal for Numerical Methods in Engineering, 2005, Vol. 64, No. 10, pp. 1350-1374.

[83] Li, Z. X., "A Co-rotational Formulation for 3D Beam Element using Vectorial Rotational Variables", Computational Mechanics, 2007, Vol. 39, No. 3, pp. 309-322

[84] Li, Z. X., and Vu-Quoc, L., "A Mixed Co-rotational 3D Beam Element Formulation for Arbitrarily Large Rotations”, Advanced Steel Construction, 2010, Vol. 6, No. 2, pp. 767-787.

[85] Li, Z. X., "Bionics and Computational Theory using Advanced Finite Element Methods", Science Press: Beijing, 2009.

[86] Tessler, A., and Hughes, T. J. R., “A 3-node Mindlin Plate Element with Improved Transverse-shear", Computer Methods in Applied Mechanics and Engineering, 1985, Vol. 50, No. 1, pp. 71-101.

[87] MacNeal, R. H., "Finite Elements: Their Design and Performance", Marcel Dekker Inc. New York, 1993, pp. 369-417. 